\title{
An integrated computational approach of molecular dynamics simulations, receptor binding studies and pharmacophore mapping analysis in search of potent inhibitors against tuberculosis
}

\author{
Shivangi Agarwal ${ }^{a}$, Ekta Verma ${ }^{a}$, Vivek Kumar ${ }^{b}$, Namrita Lall ${ }^{b}$, Samaresh Sau ${ }^{c}$, Arun K. \\ Iyer $^{\mathrm{c}, \mathrm{d}}$, Sushil K. Kashaw ${ }^{\mathrm{a}}$
}

aDepartment of Pharmaceutical Sciences, Dr. Harisingh Gour University (A Central University), Sagar, [MP]-India

${ }^{b}$ Department of Plant and Soil Sciences, University of Pretoria, South Africa

'Use-inspired Biomaterials \& integrated Nano Delivery (U-BiND) Systems Laboratory, Department of Pharmaceutical Sciences, Wayne State University, Detroit, Michigan, USA

${ }^{d}$ Molecular Therapeutics Program, Karmanos Cancer Institute, Detroit, Michigan, USA

\section{Highlights}

- Current approach was helpful to examine essential features required for ligand binding into active site of Mtb EH.

- QSAR studies revealed descriptors SssOE-index, SsssNcount, chi1, T_2_N_5, SdOcount \& k2alpha influenced activity.

- One hydrogen bond acceptor, donor, aromatic ring \& two hydrophobic groups are essential features for activity.

- Compounds with admantane ring on one side \& tri-fluorophenyl ring on other side displayed good docking scores.

- The MD simulations revealed the binding site flexibility and ligand-protein complex's binding energy.

\author{
*Corresponding author \\ Dr. Sushil Kumar Kashaw \\ Assistant Professor \\ Department of Pharmaceutical Sciences \\ Dr. Harisingh Gour University \\ Sagar (M.P.)-470003, India \\ Fax No. 07582264163 \\ E-mail: sushilkashaw @ gmail.com \\ Phone: +91-9425655720 \\ www.dhsgsu.ac.in
}




\section{Graphical abstract}

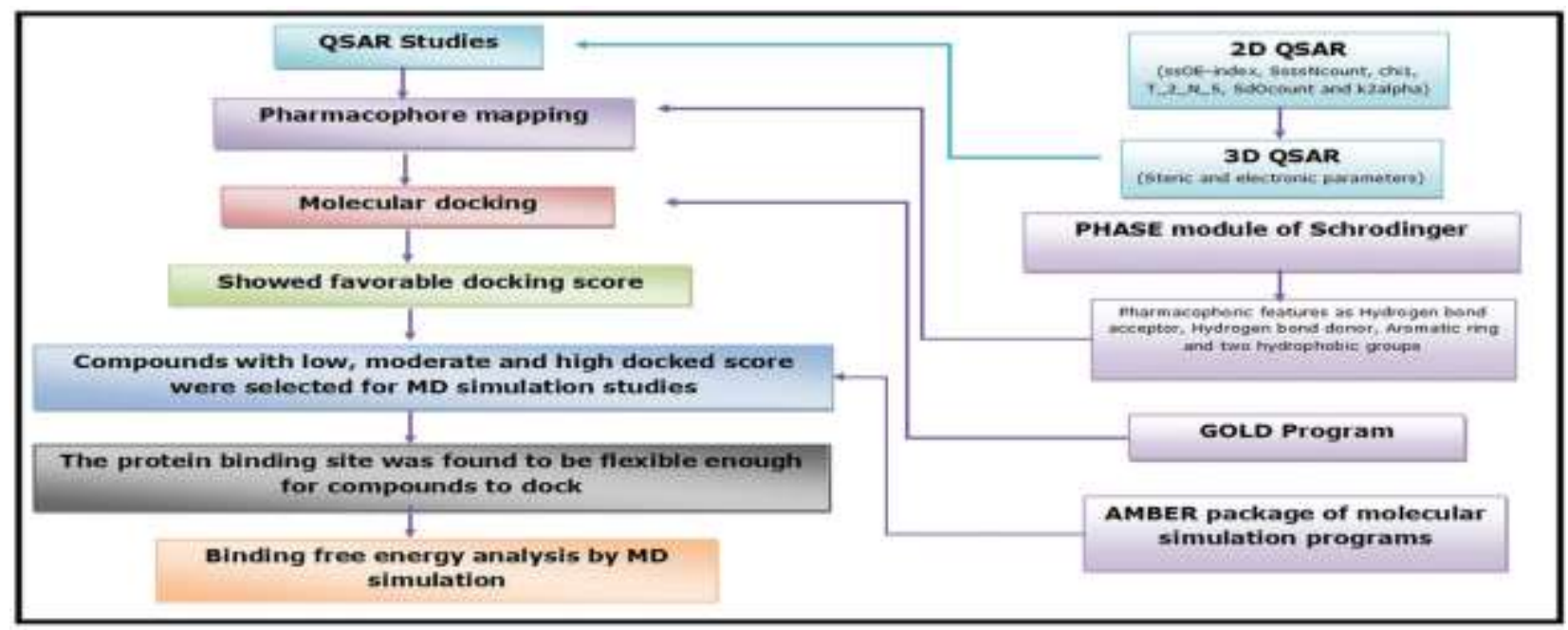

\section{Abstract}

Tuberculosis is an infectious chronic disease caused by obligate pathogen Mycobacterium tuberculosis that affects millions of people worldwide. Although many first and second line drugs are available for its treatment, but their irrational use has adversely lead to the emerging cases of multiple drug resistant and extensively drug-resistant tuberculosis. Therefore, there is an intense need to develop novel potent analogues for its treatment. This has prompted us to develop potent analogues against TB. The Mycobacterium tuberculosis genome provides us with number of validated targets to combat against TB. Study of Mtb genome disclosed six epoxide hydrolases (A to $\mathrm{F}$ ) which convert harmful epoxide into diols and act as a potential drug target for rational drug design. Our current strategy is to develop such analogues which inhibits epoxide hydrolase enzyme present in Mtb genome. To achieve this, we adopted an integrated computational approach involving QSAR, pharmacophore mapping, molecular docking and molecular dynamics simulation studies. The approach envisaged vital information about the role of molecular descriptors, essential pharmacophoric features and binding energy for compounds to bind into the active site of epoxide hydrolase. Molecular docking analysis revealed that analogues exhibited significant binding to Mtb epoxide hydrolase. Further, three docked complexes $2 \mathrm{~s}, 37 \mathrm{~s}$ and $\mathbf{1 5} \mathrm{s}$ with high, moderate and low docking scores respectively were selected for molecular dynamics simulation studies. RMSD analysis revealed that all complexes are stable with average RMSD below $2 \AA$ throughout the 10 ns simulations. The B-factor analysis showed that the active site residues of epoxide hydrolase are flexible enough to interact with inhibitor. Moreover, to confirm the binding of these urea derivatives, MM-GBSA binding energy analysis were performed. The calculations showed that $37 \mathbf{s}$ has more binding affinity $(\Delta \mathrm{Gtotal}=-52.24 \mathrm{kcal} / \mathrm{mol})$ towards epoxide hydrolase compared to $2 \mathrm{~s}$ $(\Delta \mathrm{Gtotal}=-51.70 \mathrm{kcal} / \mathrm{mol})$ and $15 \mathrm{~s}(\Delta \mathrm{Gtotal}=-49.97 \mathrm{kcal} / \mathrm{mol})$. The structural features inferred in our study may provide the future directions to the scientists towards the discovery of new chemical entity exhibiting anti-TB property.

Keywords: Molecular dynamics simulation, pharmacophore mapping, molecular docking, epoxide hydrolase, tuberculosis. 


\section{INTRODUCTION}

Tuberculosis (TB) is an infectious chronic disease caused by obligate pathogen Mycobacterium tuberculosis $(\mathrm{Mtb})$. It is contagious due to transmission of pathogen through the air. Mtb is an airborne obligate pathogen which mainly affects the upper respiratory tract. The cell wall of the bacteria is made of characteristic long chain mycolic acids and multilayered peptidoglycans and possess high lipid content due to the waxy coating on the cell surface. When infected, it reaches the lungs where they are taken up by alveolar macrophages where they grow and invade [1, 2]. The Mtb conquers mostly pulmonary regions but extra-pulmonary regions may also be affected [3]. In 2015, about 10.4 million new cases of TB and 1.4 million deaths are reported due to TB. TB remained one of top leading killer worldwide in 2015. Although the antibiotics can conquer the infectious disease but their irrational use has adversely lead to the emergence of resistance cases in TB patients. The MDR-TB (Multi-drug resistant tuberculosis) is caused by micro-organism which are resistant to treatment against rifampicin and isoniazid. The XDR-TB (extensively drug-resistant tuberculosis) is associated with resistance to rifampicin, isoniazid, one of the quinolones and one of the aminoglycosides and the total drug resistant TB (TDR-TB) is resistance to all of the first line and second line anti-TB drugs [4]. The random mutation/alteration in genes is majorly a basis of tuberculosis resistance cases [5]. The reactivation of latent infection, especially in case of HIV infected individuals leads to further challenge the success treatment therapy of TB [6]. WHO initiated DOTS therapy for the treatment of TB which includes administration of combinations of first line drugs as Isoniazid, Ethambutol, Rifampicin, Streptomycin, and Pyrazinamide. The therapy proved to be very efficient and economic [7]. But it is quite lengthy and takes about six to nine months. [8]. Moreover, it causes several adverse effects like peripheral neuritis, hepatotoxicity, ototoxicity, nephrotoxicity, cutaneous syndrome, respiratory syndrome and hyperuricemia [9].

Tuberculosis is still one of top cause of mortality all over the world. Various researchers have been putting continuous efforts for the discovery of novel scaffolds. Jadhavar et al., established SAR and synthesized the diverse library of 2-styrylquinazolones by exploring its $\mathrm{C}$ and $\mathrm{D}$ ring for different derivatives. [10] Tanwar et al., modified the quinoline nucleus by incorporating amide group in it and prepared the series of compounds by doing substitution at various position of quinoline nucleus. They found quinoline-based compounds to be more potent than standard drugs having MIC value of 0.2 and $0.39 \mu \mathrm{g} / \mathrm{ml}$ [11]. Pancholia et al., modified and designed benzo[d]thiazol-2-yl(piperazin1-yl) methanone scaffold and attached to it piperazine moiety in their laboratory by covalent bonding. Out of the synthesized compounds, about seventeen showed MIC values in 1-10 $\mu \mathrm{M}$ range when tested for anti-mycobacterial potential. [12]. Also, the oxazolidinones compounds have 
shown good anti-mycobacterial activity. The first oxazolidinones Dup-721 have shown good activity. The compounds as Linezolid, sutezolid and AZD5489 are in clinical phase evaluation. But the myelosuppression caused by this class of compounds have been a major limiting factor for their development [13]. Only two new molecules named bedaquinile and delamanid have been approved by WHO for treatment of selected TB cases over a period of fourty years. Recently, the drugs such as Carbapenems and linezolid have been repurposed for treatment of XDR-TB. In last few years, much attention has been given by WHO for treatment of TB in paediatric group. Those at a high risk of drug-drug interaction and toxicity have been monitored by therapeutic drug monitoring. However, no standard protocol has been designed to monitor and assess such type of cases [14]. The toxicity of drug is a significant health problem occurring as result of administration of a single drug dose or combination of doses. It can occur if a drug dose increases than the prescribed dose either by intention or by accident. Sometimes, it is often undetected by the doctors when symptoms such as mental retardness, memory loss, weakness, blurring in vision are not suspected to be caused by it. It is a significant concern to pharmaceutical industry when toxicity issues can lead to withdrawn of drugs from the market [15]. In between 1975 to 2000, about 548 new chemical entities have been approved by Food and Drug administration (FDA), out of which $2.9 \%$ were withdrawn from the market. Pharmacovigilance is the drug safety branch addressing the issues of adverse drug reactions and toxicities. The assessment of drug toxicity is done by calculating the risk to benefit ratio of a drug which is the ratio of risk of adverse effects associated with the drug to its potential benefit. It is calculated as the ratio of lethal dose to the effective dose $\left(\mathrm{LD}_{50} / \mathrm{ED}_{50}\right)$. The higher the ratio, the safer the drug [16]. Various investigations as the measurement of plasma drug concentration, urine drug concentration, tissue concentrations, biopsies etc are means of addressing and monitoring these adverse effects. Further, the knowledge of human genome can help us to predict the action of drugs including the adverse effects, leading to a possibility of safe and effective therapy to a patient [17].

In the present scenario, it has become a challenge to discover appropriate therapeutic regimen for TB. [8]. Moreover, the genome of Mtb serve as a platform and provides with hundreds of validated targets to design and discover novel agents against TB. The epoxide hydrolase (EH) enzyme was found to present in genome of Mtb which serves as a platform for rational drug design $[18,19]$. The compounds containing epoxide moiety are highly toxic and destructive for mycobacterium [20]. The Mtb EH plays a significant role in detoxification and metabolizes toxic epoxides into non-toxic 1,2-diols via dihydroxylation reaction. [19]. Therefore, EH act as a potential drug target against tuberculosis. The carbanilide (1,3-disubstituted urea derivatives) inhibit EH and 
expected to be bactericidal and was found to show an $\mathrm{IC}_{50}$ value of $19 \mu \mathrm{M}$ against Mtb epoxide hydrolase B. It therefore acts as potential lead compound to develop and design novel effective analogues to battle against TB [18].

The computational aided drug design (CADD) based approaches play a remarkable role in drug design and discovery. The approach provides a crucial guidance and directs us towards rational drug design. It hastens speed, efficacy and minimize the time consumption and total expenditure associated, therefore facilitating the process of drug discovery [21, 22]. Molecular docking is one of the computational based approaches that predicts the binding orientation of the inhibitor compounds with the protein structure. It explores the active site amino acid residues which are significant for ligand binding. The GOLD (Genetic optimization ligand docking) is a genetic algorithm based docking program [23]. Its goal is to achieve an optimized conformation for both ligand and receptor. [23, 24]. Molecular dynamics (MD) simulation is most important computational approach which predicts the behavior of protein ligand complex as a function of time. It provides insight into the atomic motions of individual particles. [25, 26]. In continuation of our earlier work and continuous efforts on various CADD techniques in the field of drug design and discovery, [27-32], a combined approach of quantitative structure activity relationships (QSAR), pharmacophore mapping, molecular docking and molecular dynamics simulation has been applied to the reported urea analogues against epoxide hydrolase. The approach was very helpful to probe essential features required for ligand binding into the binding site of epoxide hydrolase, active site amino acids residues involved in binding, binding free energy changes and per-residue contribution towards binding. The goal of our present study is to develop a hypothesis which states important structural requirements for ligands to bind into the active site of epoxide hydrolase enzyme and is helpful in drug design against TB. In the first part of our study, we present SAR studies of compounds containing urea moiety based on 2D-QSAR, 3D-QSAR and pharmacophore mapping analysis and then in second part, we present molecular docking of these compounds into the binding pocket of epoxide hydrolase. In addition, the docked complex obtained by molecular docking with high, moderate and low docking scores were analyzed for stability of complex, protein binding site flexibility, binding free energy and per residue energy contribution in binding by using molecular dynamics simulations approach. 


\section{MATERIALS AND METHODS}

\subsection{Selection of data}

A set of 40 molecules with 1,3-disubstituted urea derivatives [33] was subjected to the QSAR analysis (2D-QSAR and 3D-QSAR), pharmacophore mapping and molecular docking. Further, the selected molecules were employed for molecular dynamics simulation analysis. All QSAR studies were performed by VLife Molecular design suite, version 4.4 [34]. Phamacophore and docking analysis was done by the PHASE, version 9.3 [35] and GOLD program, version 5.2. [36] respectively. Molecular dynamics simulation studies were performed using AMBER software [37] All the reported compounds and their corresponding biological activities are represented in Table 1. The workflow for the employed approach is shown in figure 1.

Table 1. Structural detail of the compounds in the series along with their biological activity

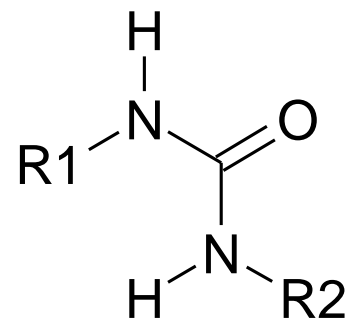

\begin{tabular}{|c|c|c|c|}
\hline Compound & R1 & $\mathrm{R} 2$ & $\mathrm{IC}_{50}(\mathrm{nM})$ \\
\hline $1 \mathrm{~s}$ & (2-Adamantyl) & (2,3,4-trifluorophenyl & 0.4 \\
\hline $2 s$ & (1-Adamantyl) & (2,3,4-trifluorophenyl) & 0.4 \\
\hline $3 \mathrm{~s}$ & (1-Adamantyl)methyl) & (2,3,4-trifluorophenyl & 0.4 \\
\hline $4 s$ & Heptyl & (2,3,4-trifluorophenyl) & 0.4 \\
\hline $5 s$ & (2,3,4-Trifluorophenyl) & $\begin{array}{l}\text { (2,6,6-trimethylbicyclo[3.1.1]-heptan-3- } \\
\text { yl }\end{array}$ & 0.4 \\
\hline $6 s$ & $\begin{array}{l}\text { ((6,6-Dimethylbicyclo[3.1.1]heptan-2- } \\
\text { yl)methyl) }\end{array}$ & (2,3,4-trifluorophenyl) & 0.4 \\
\hline $7 \mathrm{~s}$ & Cyclooctyl & (2,3,4-trifluorophenyl) & 0.4 \\
\hline $8 \mathrm{~s}$ & Cyclohexyl & (2,3,4-trifluorophenyl) & 1.2 \\
\hline $9 \mathrm{~s}$ & Cyclopentyl & (2,3,4-trifluorophenyl) & 56.2 \\
\hline $10 \mathrm{~s}$ & (2-Adamantyl) & (4-cyanophenyl & 1.1 \\
\hline $11 \mathrm{~s}$ & (2-Adamantyl) & phenethyl & 4.5 \\
\hline $12 \mathrm{~s}$ & (3-Acetylphenyl) & (2-adamantyl) & 0.5 \\
\hline $13 \mathrm{~s}$ & (2-Adamantyl) & benzyl & 14.8 \\
\hline $14 \mathrm{~s}$ & (2-Adamantyl) & (3-chloro-2-methylphenyl) & 4.5 \\
\hline
\end{tabular}




\begin{tabular}{|c|c|c|c|}
\hline $15 \mathrm{~s}$ & (2-Adamantyl) & isopropyl & 84.4 \\
\hline $16 \mathrm{~s}$ & (2-Adamantyl) & tert-butyl & 24.2 \\
\hline $17 \mathrm{~s}$ & (2-Adamantyl) & propyl & 61.4 \\
\hline $18 \mathrm{~s}$ & (2-Adamantyl) & cyclohexyl & 0.4 \\
\hline $19 \mathrm{~s}$ & (2-Adamantyl) & pentyl & 6.4 \\
\hline $20 \mathrm{~s}$ & (2-Adamantyl) & hexyl & 1.0 \\
\hline $21 \mathrm{~s}$ & (2-Adamantyl) & heptyl & 0.5 \\
\hline $22 s$ & (1-(1-Adamantyl)methyl) & (3-chloro-4-methylphenyl) & 0.4 \\
\hline $23 \mathrm{~s}$ & (3-Chloro-4-methylphenyl) & heptyl & 1.6 \\
\hline $24 \mathrm{~s}$ & (3-Chloro-4-methylphenyl) & cyclooctyl & 0.4 \\
\hline $25 \mathrm{~s}$ & (3-Chloro-4-methylphenyl) & (3-fluorobenzyl) & 16.6 \\
\hline $26 \mathrm{~s}$ & (3-Chloro-4-methylphenyl) & (4-phenylbutan-2-yl)- & 15.0 \\
\hline $27 \mathrm{~s}$ & (2-Fluoro-3-(trifluoromethyl)phenyl) & heptyl & 0.4 \\
\hline $28 \mathrm{~s}$ & Cyclooctyl & (2-fluoro-3-(trifluoromethyl)phenyl) & 0.4 \\
\hline $29 \mathrm{~s}$ & (2-Fluoro-3-(trifluoromethyl)phenyl) & (4-phenylbutan-2-yl) & 1.9 \\
\hline $30 \mathrm{~s}$ & (3-Chlorobenzyl) & (2-fluoro-3-(trifluoromethyl)-phenyl) & 3.7 \\
\hline $31 \mathrm{~s}$ & (2-Adamantyl) & (2,3,4-trifluorophenyl)thiourea & 4.8 \\
\hline $32 \mathrm{~s}$ & 2-Adamantyl & 2,3,4-trifluorophenylcarbamate & 463.8 \\
\hline $33 \mathrm{~s}$ & (2-Adamantyl) & 3-methyl-3-(2,3,4-trifluorophenyl) & 38.1 \\
\hline $34 \mathrm{~s}$ & (2-Adamantyl)-1,3-dimethyl & (2,3,4-trifluorophenyl) & 1838.1 \\
\hline $35 \mathrm{~s}$ & (2-Adamantyl) & (4-methoxyphenyl) & 14.3 \\
\hline $36 s$ & Methyl 4-(3-(1-adamantyl)ureido) & -2-hydroxybenzoate & 1.2 \\
\hline $37 \mathrm{~s}$ & (1-Adamantyl) & (2-ethoxyethyl) & 524.8 \\
\hline $38 \mathrm{~s}$ & (1-Adamantyl) & (3-methoxypropyl) & 386.1 \\
\hline $39 \mathrm{~s}$ & (1-Adamantyl) & (2-isopropoxyethyl) & 260.4 \\
\hline $40 \mathrm{~s}$ & (1-Adamantyl) & (2-propoxyethyl) & 242.6 \\
\hline
\end{tabular}




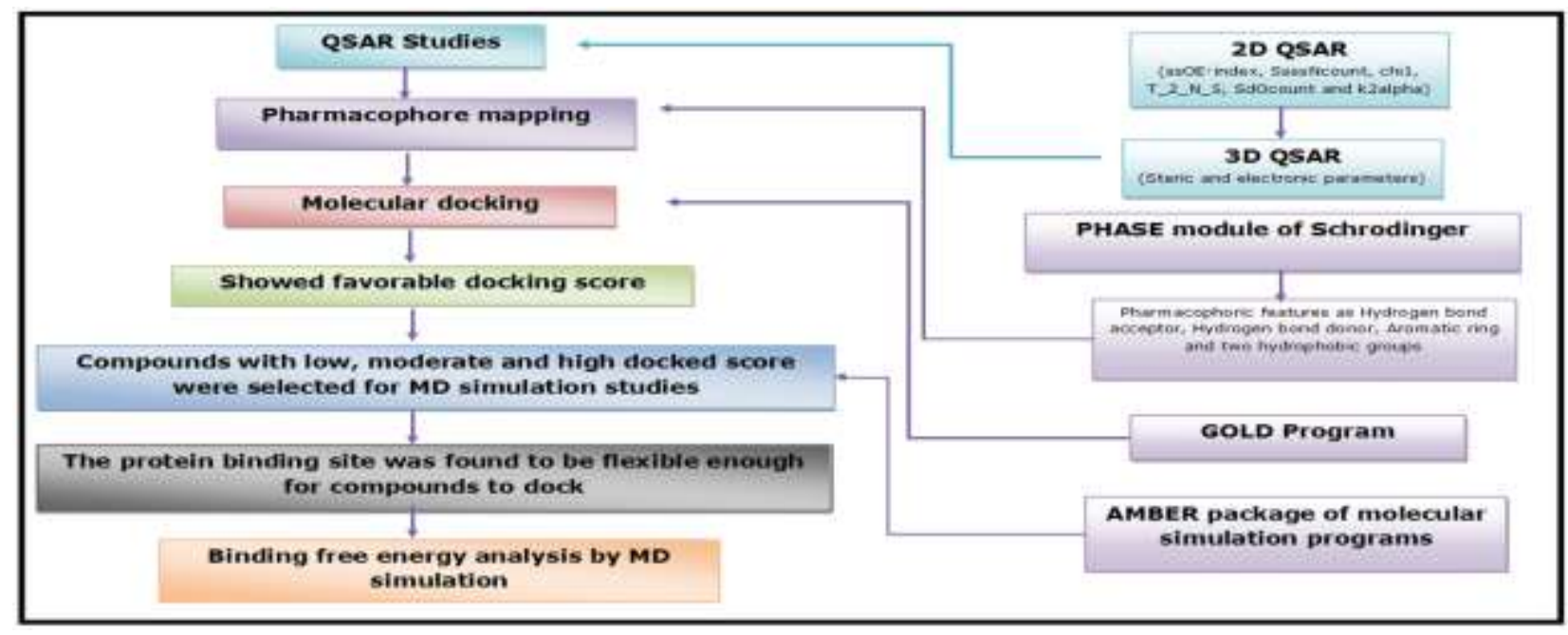

Fig.1 Workflow of the employed computational approach

\subsection{Preparation of molecules for 2D-QSAR and 3D-QSAR analysis}

The molecules were sketched using Chemdraw 8.0 and optimized by Molecular Mechanics Force Field (MMFF) method. The molecules were subjected to 2D-QSAR and 3D-QSAR analysis and the validated model was developed for each of the analysis. The developed model set up a relationship between dependent and independent variables [38].

\subsubsection{D-QSAR and 3D-QSAR analysis}

For 2D-QSAR, the compounds were divided into training and test set by manual selection method. The test set covers $1 / 5$ th of total molecules and was chosen within minimum-maximum range of training set. The uni-column statistics was performed for test and training set. The 2D-QSAR model was built by PLS (Partial Least Square) regression method using forward-backward as a variable selection method. The descriptors such as topological, physico-chemical and alignmentindependent were computed by 2D-QSAR. [39]. The 3D-QSAR examines the electrostatic and steric regions present in the molecule against the biological response and quantitate the relationship between structural features and biological activity. For 3D-QSAR, the structures were aligned by template based alignment method using a reference molecule (Figure 2a) and a template (Figure $2 \mathrm{~b}$ ). The regression analysis was performed by $\mathrm{kNN}$ (k-nearest neighbour) method. The alignment of molecules is shown in figure 3.[40]. 

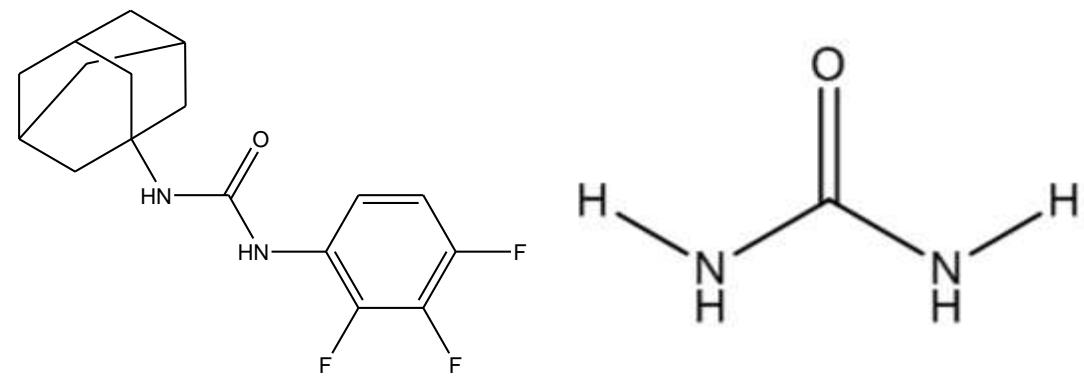

Fig.2. Reference molecule (comp2s) (a) and urea substructure template (b) for alignment

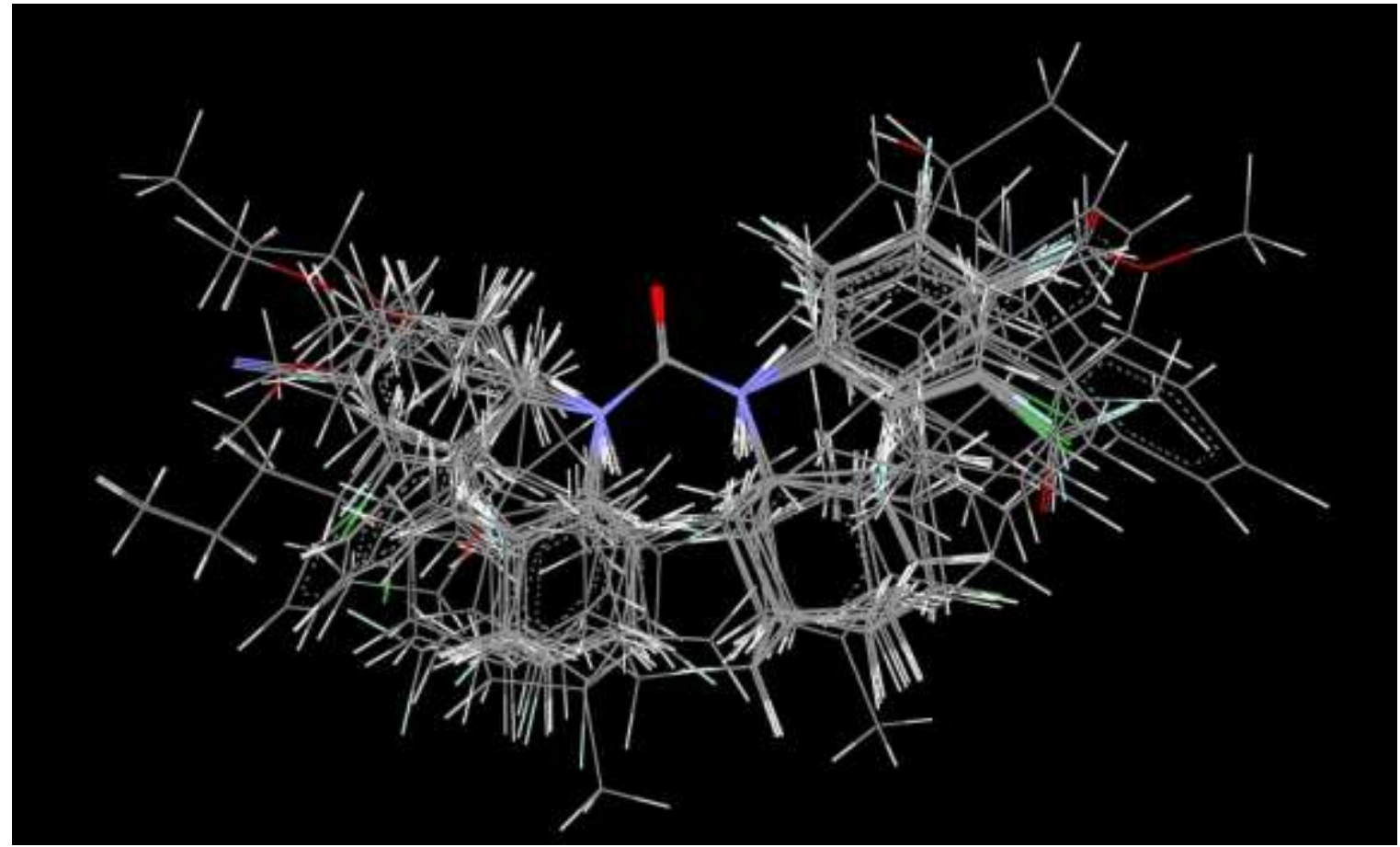

Fig.3. Aligned molecules by template based alignment method

\subsection{Pharmacophore Mapping}

In addition to set up the relationship between molecular descriptors and biological activity, we identified the important pharmacophoric features which are essential for biological activity by pharmacophore mapping using PHASE module. The PHASE module generated various pharmacophore hypotheses and computed their survival scores. Among those, the best one was selected containing the essential features responsible for the activity.

\subsubsection{Preparation of ligands}

The molecules were refined using 'LigPrep' option provided by PHASE. The hydrogen atoms were added to complete the valency of the molecules, converted to 3D and optimized. The conformers 
were generated by Monte Carlo Molecular Mechanics (MCMM) method which were then employed for pharmacophore hypotheses generation [41].

\subsubsection{Pharmacophore hypotheses generation}

Common pharmacophoric features were identified and the pharmacophore hypotheses were created. The hypotheses were examined using a scoring function as survival score. The site, vector, volume and selectivity scores, $\mathrm{p}$-value and number of matches were computed for each of the generated hypotheses. The best hypothesis was selected among them based on the scoring values. The regression analysis was then performed by PLS method and pharmacophore-based 3D-QSAR model was generated for the hypotheses [42].

\subsection{Molecular Docking analysis}

To find out the binding interaction of compounds containing essential pharmacophoric features, we performed their molecular docking with epoxide hydrolase enzyme. To perform molecular docking, the crystal structure of epoxide hydrolase enzyme in complex with diphenylurea (Figure 4) with resolution of $2.4 \AA$ was obtained from the protein data bank having PDB ID-2ZJF with R-value work and R-value free as 0.202 and 0.256 respectively $[18,43]$. The molecular docking was performed by GOLD program. The hydrogens were added to crystal structure of protein and cocrystallized ligand was extracted from its binding pocket. The compounds (Table 1) were docked by keeping the default GA run as 10 . The GA runs specify the number of times each ligand is to be docked. The CHEMPLP (Piecewise Linear Potential) has been found to give highest success rates and therefore used as scoring function to rank docked compounds in relation to each other. The docking was performed using the standard default settings as population size of 100, selection pressure of 1.1, no. of operation as 100000, no. of islands as 5 and niche size of $2[44,45]$. 


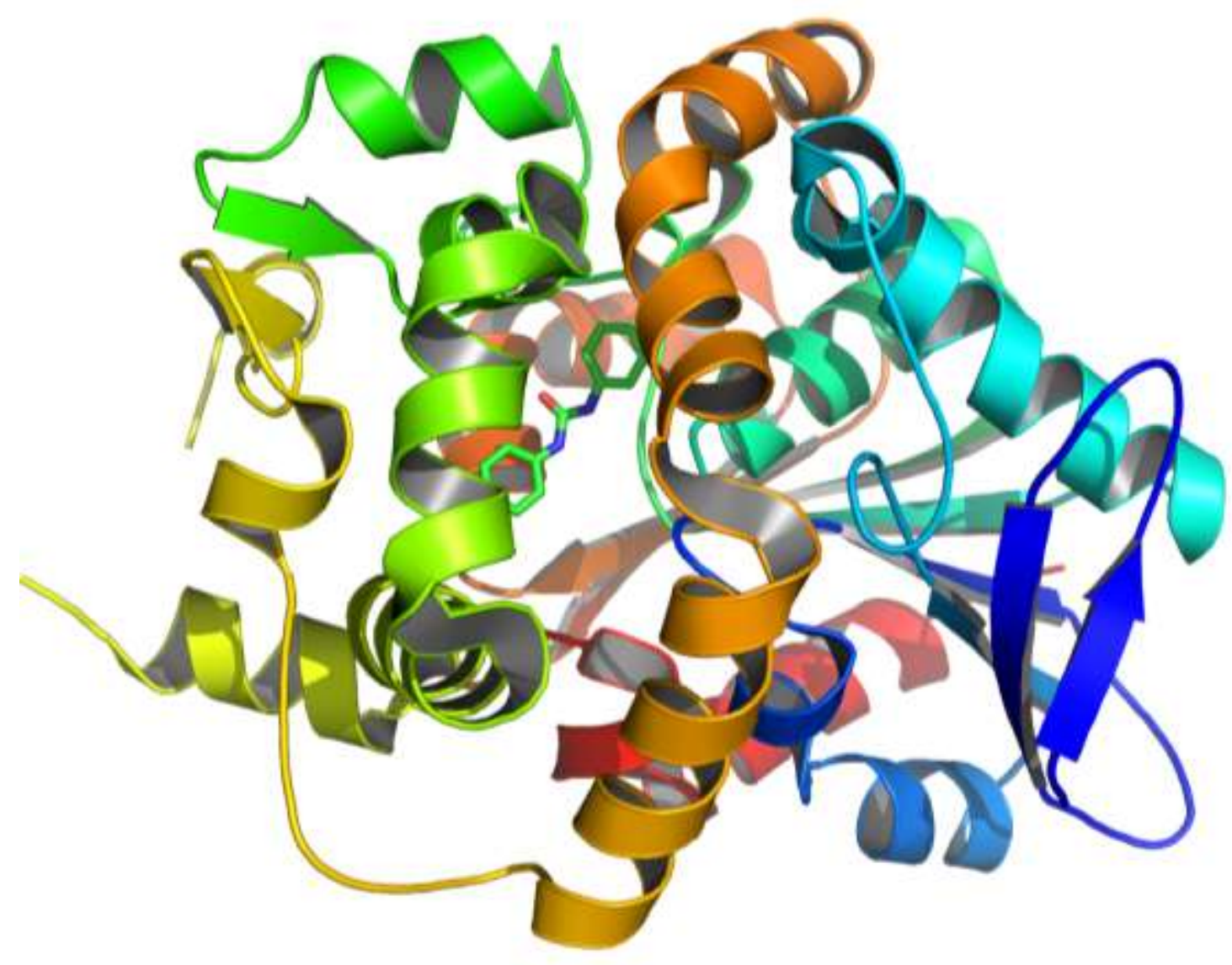

Fig.4. 3D view of Mtb epoxide hydrolase (PDB ID-2ZJF)

\subsection{Molecular dynamics simulations}

\subsubsection{Data Selection}

The complex systems were prepared using molecular docking protocol mentioned in the previous section. The molecule $2 \mathrm{~s}$ has highest docking score while $37 \mathrm{~s}$ and $15 \mathrm{~s}$ have moderate and low docking scores respectively. The docked binary complexes comp_2s, comp_15s and comp_37s i.e. epoxide hydrolase (PDB ID: 2ZJF) in complex with molecules $2 \mathrm{~s}, 15 \mathrm{~s}$ and $37 \mathrm{~s}$ were considered for molecular dynamics (MD) simulations studies.

\subsubsection{System preparation for molecular dynamics simulations}

The system preparation methodology is adopted as discussed by Kumar et al. [46, 47] The preparation of the topology and coordinate files for three systems was carried out using complexes comp_2s, comp_15s and comp_37s. The epoxide hydrolase enzyme was corrected for atoms and bond orders followed by energy minimization to potentially relax the structures. FF14SB force field 
parameters were applied for the protein using the AMBER (Assisted Model Building with Energy Refinement) tLeaP module [48]. The ligands $2 \mathbf{s}, \mathbf{1 5} \mathrm{s}$ and $37 \mathrm{~s}$ were extracted and corrected for atom types using Discover Studio 2.5 [49]. The parameters missing for the ligands were generated using the general amber force field (GAFF) and the Parmchk module of Antechamber [50, 51]. The prepared complex systems were solvated with TIP3P water model by creating an isometric water box, where distance of the box was set to $10 \AA$ from periphery of protein [52]. The solvated complex systems were then neutralized through the AMBER LeaP module by adding necessary amount of counter ions $(\mathrm{Na}+)$ to construct the system in electrostatically neutral positions. The whole assembly was then saved as per the requirement of free energy calculations. It involved preparing the parameter and coordinate files for the complex, protein and the ligand without solvation. Further, the prepared topology and coordinate files of solvated complexes were used as input for sander module of the AMBER16 [37]. The optimization and relaxation of solvent and ions were performed by means of two energy minimization cycles using 1500 and 2000 steps. The initial 1000 steps of each minimization cycle were performed using steepest descent followed by conjugate gradient minimization for rest of the steps.

In the first part of minimization, epoxide hydrolase-ligand complex was kept fixed to allow water and ion molecules to move, followed by the minimization of the whole system (water, ions and complex) in the second part. Heating was performed using a NVT ensemble for 20 ps where ATPase-ligand complex was restrained with a very small force constant of $5 \mathrm{kcal} / \mathrm{mol} / \AA 2$. The temperature was allowed to increase till $300 \mathrm{~K}$. The system was further equilibrated under constant pressure at $300 \mathrm{~K}$ for the period of $100 \mathrm{ps}$ without restrain on the complex. Final simulations i.e. production phase was performed for $10 \mathrm{~ns}$ on NPT ensemble at $300 \mathrm{~K}$ temperature and $1 \mathrm{~atm}$ pressure. The step size of 2 fs was kept for whole simulation study. Langevin thermostat and barostat were used for temperature and pressure coupling. SHAKE algorithm was applied to constrain all bonds containing hydrogen atoms [53].

Non-bonded cut-off was kept on $10 \AA$ and long range electrostatic interactions were treated by Particle Mesh Ewald method (PME) with fast Fourier transform grid spacing of approximately $0.1 \mathrm{~nm}$ [54]. Trajectory snapshots were taken at each $10 \mathrm{ps}$ of the production phase, which were used for final analysis. The minimization, heating and equilibration were performed by sander module of AMBER16, while production simulation was performed using Pmemd program of AMBER16 running on NVIDIA Tesla K20c GPU work station [55]. The production run was considered for the analysis which was carried out using the Ptraj module of the AMBER16 and VMD [56, 57]. 


\subsubsection{Root-mean-square deviation (RMSD) and B-factor}

RMSD and B-factor, were calculated using the ptraj analysis tool in the AMBER program. RMSD is the measure of the average distance between the atoms (usually the backbone atoms) of superimposed protein structures [58]. The equation below illustrates the rmsd:

$$
\mathrm{RMSD}=\frac{1}{N} \sum_{i}^{N} \delta_{i}^{2}
$$

where $\delta$ is the distance between $\mathrm{N}$ pairs of equivalent atoms.

To compare the flexibility of the structures, B-factor or thermal factor was used to calculate the mobility of the residues present in the ATP lid. It was calculated from the mean square fluctuations (msf) using the following equation:

$$
\text { Thermal factor or } B-\text { factor }=[(8 \pi * * 2) / 3](m s f)
$$

B-factor was calculated using the equation 2 for each residue and plotted against each residue [59].

\subsubsection{MM-GBSA binding free energy ( $\triangle G)$ calculations}

The binding free energy calculations were performed for all complex systems using MM-GBSA (Molecular Mechanics-Generalized Born Surface Area) considering single trajectory approach. The various previous study find this approach useful in estimation of binding free energy [60, 61]. Binding free energy calculations were performed on production phase considering last 2 ns of 10 ns run using the Born implicit solvent model of $2(\mathrm{igb}=2)$.

$$
\Delta G_{\text {Binding }}=G_{\text {Complex }}-G_{\text {Protein }}-G_{\text {Ligand }}
$$

The calculations include various energy components consisting of molecular mechanical energy ( $\left.\mathrm{E}_{\mathrm{MM}}\right)$ and polar contribution $\left(\mathrm{G}_{\mathrm{GB}}\right)$ towards solvation energy calculated by generalized Born $(\mathrm{GB})$ method respectively (Eq. 7). G $\mathrm{G}_{\mathrm{SA}}$ is the contribution from nonpolar terms towards solvation energy, and TS is the entropic contribution of the inhibitor. $\mathrm{E}_{\mathrm{MM}}$ was obtained by summing contributions from, electrostatic energy $\left(E_{\text {ele }}\right)$, VDW energy $\left(E_{\mathrm{vdw}}\right)$, and internal energy including bond, angle, and torsional angle energy $\left(\mathrm{E}_{\mathrm{int}}\right)$ using the same force field as that of MD simulations (Eq. 8).

$$
\begin{gathered}
G=E_{M M}+G_{G B}+G_{S A}-T S \\
\mathrm{E}_{\mathrm{MM}}=\mathrm{E}_{\mathrm{vdw}}+\mathrm{E}_{\mathrm{ele}}+\mathrm{E}_{\mathrm{int}}
\end{gathered}
$$

\subsubsection{The binding free energy decomposition analysis}

Free energy was decomposed to estimate the contribution of each residue in the ligand binding process and was performed by using MM-GBSA method[62]. Energy of each residue-ligand interaction is given by following equation: 


$$
\Delta G_{\text {residue-ligand }}=\Delta E_{\text {ele }}+\Delta E_{v d W}+\Delta G_{G B}+\Delta G_{S A}
$$

Where, $\Delta \mathrm{G}_{\mathrm{GB}}$ is the polar group contribution to the solvation free energy calculated using $\mathrm{GB}$ model. $\Delta \mathrm{G}_{\mathrm{SA}}$ is the non-polar group contribution to the solvation free energy calculated using ICOSA method. Similar to the binding free energy, the decomposition energy was also averaged over 200 frames taken at the interval of $100 \mathrm{ps}$ over the last $2 \mathrm{~ns}$ of $10 \mathrm{~ns}$ production run. In addition, average structures for comparison of trajectories were also obtained using the 200 frames over the last $2 \mathrm{~ns}$ of $10 \mathrm{~ns}$ simulations.

\section{RESULTS AND DISCUSSION}

The goal of our present study is to develop a hypothesis which is helpful in anti-TB drug development. The goal is achieved by employing integrated computational approach involving techniques as QSAR, pharmacophore mapping, molecular docking and molecular dynamics simulation studies. In the first part of our study, we present SAR (structure activity relationships) studies for compounds containing urea moiety and then in second part, we present molecular docking of these compounds into the binding pocket of epoxide hydrolase. The selected compounds were further analyzed for stability, protein binding site flexibility and binding free energy by using molecular dynamics simulations analysis.

\subsection{D-QSAR analysis}

The 2D-QSAR model was developed by PLS method and the best 2D model was selected based on regression coefficient values and number of outlier compounds. The biological activity equation indicating the physico-chemical parameters coefficient and their correlation with the biological activity was constructed. The 2D model showed a $\mathrm{r}^{2}$ value of 0.827 and standard error of 0.4346 (Table 2). The stability of the model was judged by leave-one-out procedure and found to be fairly good $\left(\mathrm{q}^{2}=0.8101\right)$ implying that the model would be fruitful for significant predictions. The pred_ $\mathrm{r}^{2}$ value of 0.9559 indicated the complimentary potential of the developed model. 
Table 2. Statistical results of 2D-QSAR equation generated by PLS method

\begin{tabular}{|c|c|c|c|}
\hline \multirow[t]{2}{*}{ Equation } & \multicolumn{3}{|c|}{$\begin{array}{c}\mathrm{pIC}_{50}=-0.4705 \mathrm{SssOE}-\mathrm{index}-2.1397 \mathrm{SsssNcount}+0.5987 \text { chi1 }-0.5920 \\
\text { T_2_N_7+ } 0.9016 \mathrm{SdO} \text { count }+0.2131 \mathrm{~K} 2 \mathrm{alpha}+0.6631\end{array}$} \\
\hline & $\mathrm{N}=32$ & Degree of freedom $=26$ & $\mathrm{~F}$ test $=35.6552$ \\
\hline Statistics & $\mathrm{r}^{2}=0.8727$ & $q^{2}=0.8101$ & pred_r ${ }^{2}=0.9559$ \\
\hline & $\mathrm{r}^{2} \mathrm{se}=0.4346$ & $\mathrm{q}^{2} \mathrm{se}=0.5309$ & pred_r ${ }^{2} \mathrm{se}=0.2571$ \\
\hline
\end{tabular}

The descriptors such as SssOE-index, SsssNcount, chi1, T_2_N_5, SdOcount and k2alpha were found to contribute to the biological activity. These descriptors are described as: Sss $\boldsymbol{O E}$-index: Electrotopological state indices for number of oxygen atom connected with two single bonds. SsssNcount: Defines the total number of nitrogen connected with three single bonds. Chil: Signifies retention index derived from gradient retention times. $\boldsymbol{T} \_\mathbf{2} \boldsymbol{N}_{-} \mathbf{7}$ : This is the count of number of the double bonded atoms separated from nitrogen atom by 7 bonds. SdOcount: Defines the total number of oxygen connected with one double bond. K2alpha: Signifies second alpha modified shape index. The descriptors such as chi1, SdOcount and k2alpha have their role in enhancing the activity and the descriptors such as SssOE-index, SsssNcount and T_2_N_7 decrease the activity. The correlation matrix was calculated and given in Table S1 and the values less than 0.6 indicate the absence of multi-colinearities in the model. The contribution chart indicates the $\%$ contribution of descriptors towards biological activity (Figure 5). The uni-column statistical analysis was performed to evaluate the selection criteria for compound in training and test set (Table S2). The predicted activity values were computed for each of the compounds (Table S3) and the graph was plotted between the actual and predicted biological activity values which showed a good correlation $\left(\mathrm{R}^{2}=0.9227\right)($ Figure 6). 


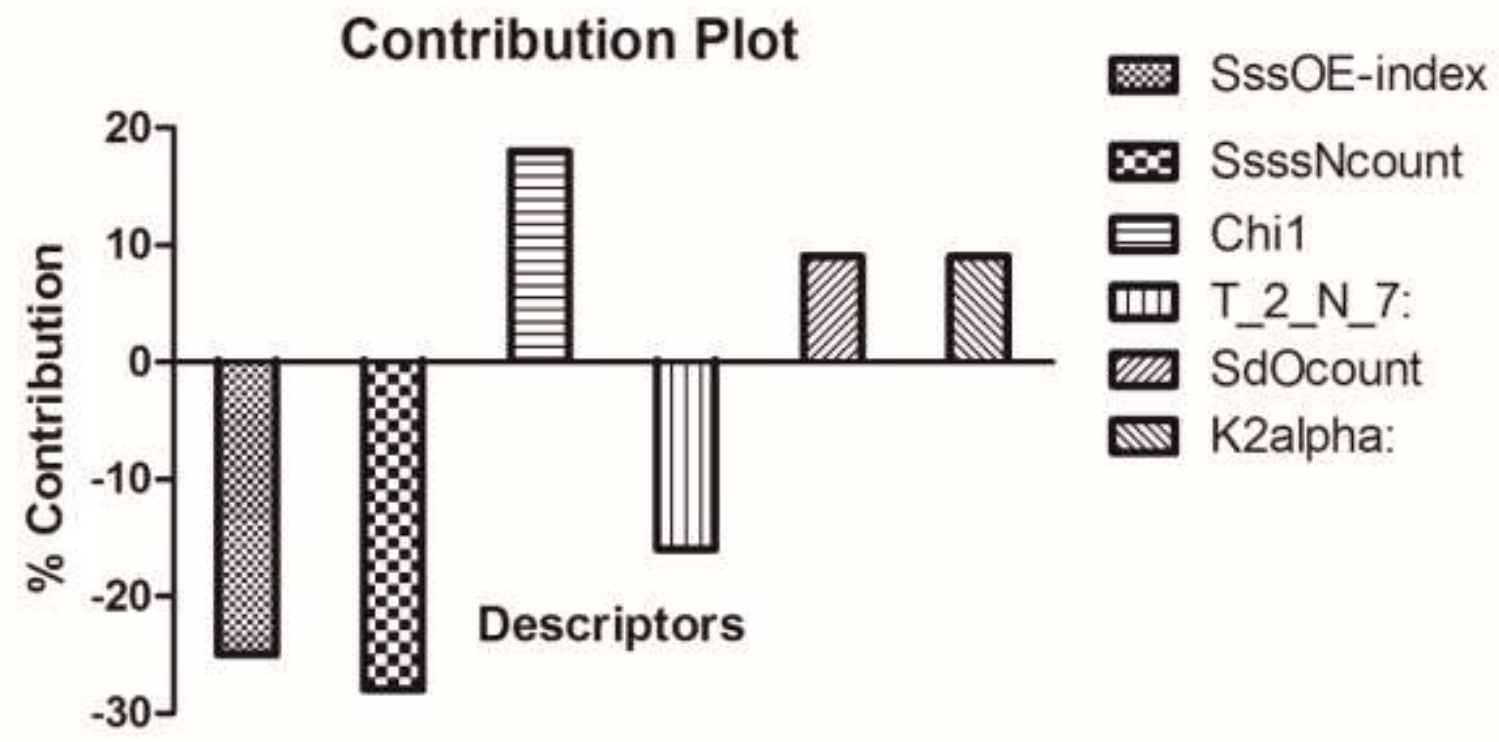

Fig. 5. Contribution chart of selected 2D descriptors towards antitubercular activity Correlation plot

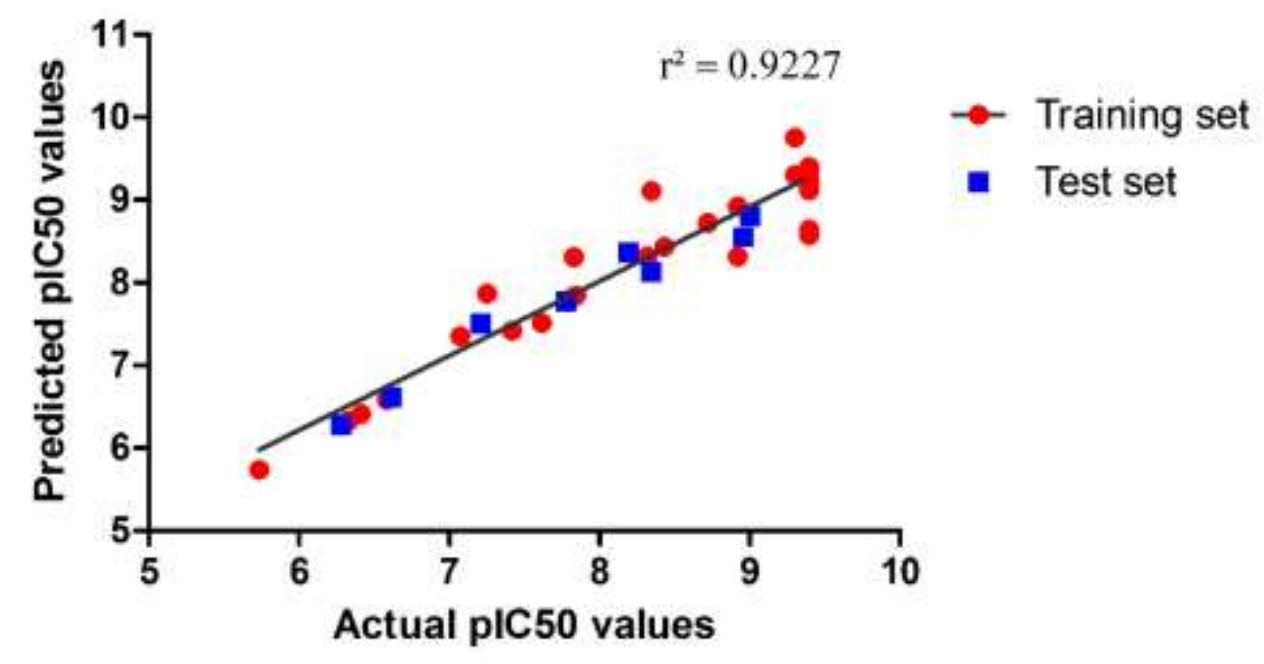

Fig. 6. Correlation plot between actual and predicted pIC50 values by 2D-QSAR

\subsection{D-QSAR analysis}

In addition to finding out the contribution of physico-chemical descriptors towards biological activity as mentioned in the above section, we estimated the contribution of steric and electrostatic parameters towards the biological activity by 3D-QSAR analysis using VLife. The 3D-QSAR model was generated by kNN-MFA analysis (k nearest neighbour molecular field analysis) method. The steric interaction energies were calculated at the lattice points of the grid using a methyl probe of +1 charge. The steric and electrostatic data points generated by 3D-QSAR analysis are shown in figure 7 . 


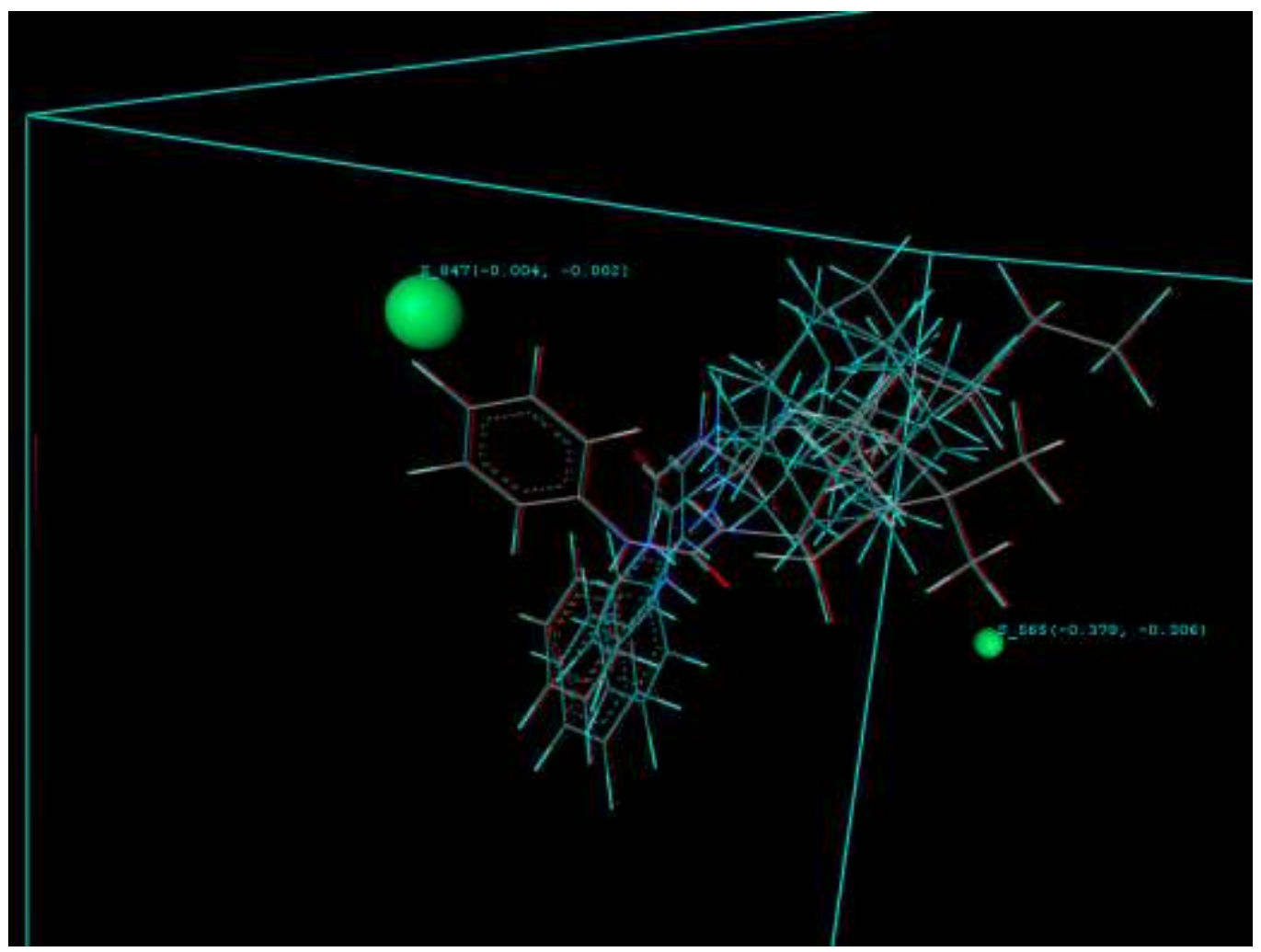

Fig. 7. Relative positions of the steric and electrostatic fields

The best model showed a cross-validated correlation coefficient i.e $\mathrm{q}^{2}$, pred_r ${ }^{2}$ and $\mathrm{K}$ nearest neighbour values of $0.8105,0.7015$ and 2 respectively. The computed parameter values are shown in Table 3. The predicted activity values were computed for each of the compounds (Table S4) and the graph was plotted between the actual and predicted biological activity values which showed a correlation value $\mathrm{R}^{2}=0.7826$ (Figure 8 ). The ranges of data point values were based on the variation of the field values at the chosen points using the most active molecule and its nearest neighbour set. Points generated in kNN-MFA 3D-QSAR model are S_565 (-0.378, -0.306), S_847 (-0.004, 0.002) i.e. steric data points at lattice points 565 and 847 respectively. Negative values of steric field descriptors indicated the requirement of negative steric potential, for enhancing the biological activity of urea derivatives against Mtb EH. The negative value of steric factors indicates the preference of less steric substituents at generated data points S_565 (-0.378, -0.306) and S_847 ($0.004,-0.002$ ) around urea pharmacophore. 
Table 3. Statistical results of 3D-QSAR model generated by kNN MFA method

\begin{tabular}{|c|}
\hline Model summary \\
\hline kNN Method \\
\hline Training Set Size $=31$, Test Set Size $=7$ \\
\hline Statistics: \\
\hline $\mathrm{k}$ Nearest Neighbour $=2, \mathrm{n}=31$ \\
\hline Degree of freedom $=27$ \\
\hline $\mathrm{q}^{2}=0.8105, \mathrm{q}^{2} \_\mathrm{se}=0.4662$ \\
\hline Pred_r ${ }^{2}=0.7015$, pred_r ${ }^{2}$ se $=0.7331$ \\
\hline Selected descriptor \& their range: \\
\hline S_565 $(-0.378,-0.306)$ \\
\hline S_847 $(-0.004,-0.002)$ \\
\hline
\end{tabular}

\section{Correlation plot}

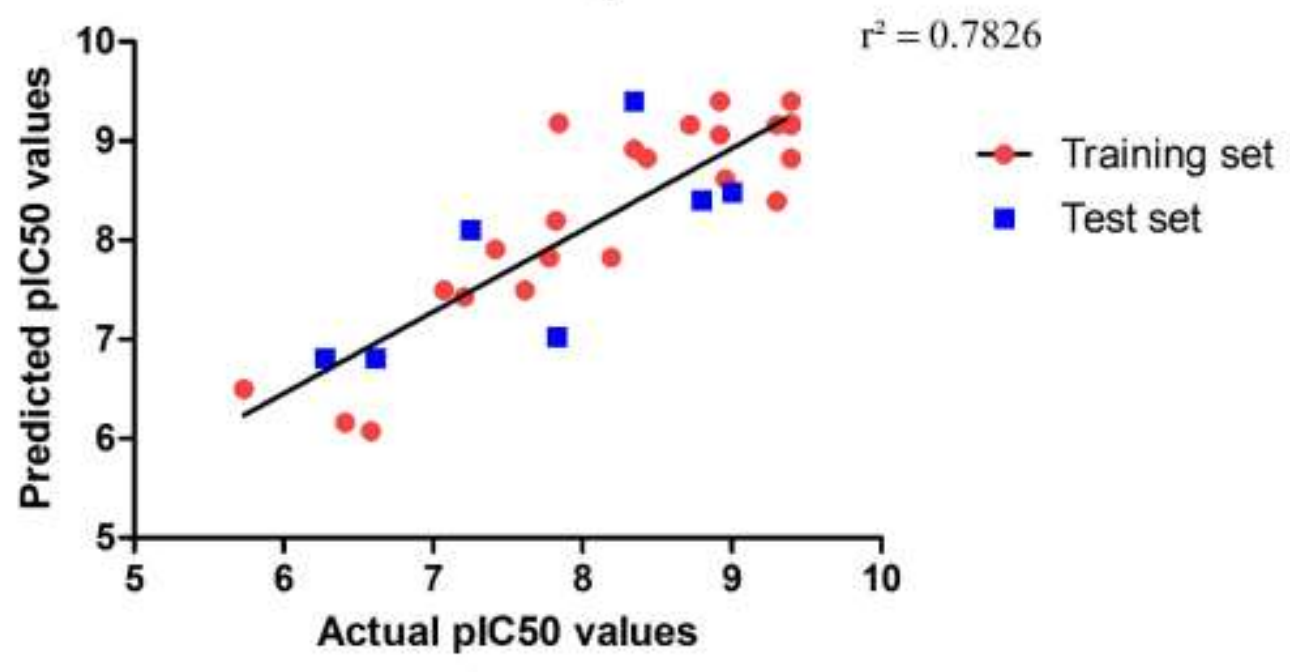

Fig. 8. Correlation plot between actual and predicted pIC50 values by 3D-QSAR

\subsection{Pharmacophore mapping}

After finding out the role of physico-chemical, steric and electronic descriptors in biological activity by QSAR, we identified common features which are essential for biological activity. For the same reason, we generated pharmacophore hypotheses. The total of four pharmacophoric hypotheses were developed and their scoring function as survival scores and other as site, vector, volume and selectivity score are represented in Table 4. The top model comprised of five features hypothesis, 
i.e. ADHHR.27 which include one acceptor group (A), one donor group (D), two hydrophobic groups $(\mathrm{H})$ and one aromatic ring $(\mathrm{R})$. This is denoted as A1D2H5H6R8.

Table 4. Scoring results of the different pharmacophore hypotheses

\begin{tabular}{ccccccccccc}
\hline S.No. & ID & Survival & $\begin{array}{c}\text { Survival - } \\
\text { inactive }\end{array}$ & $\begin{array}{c}\text { Post- } \\
\text { hoc }\end{array}$ & Site & Vector & Volume & Selectivity & \# Matches \\
\hline 1 & ADHHR.27 & 3.297 & 1.676 & 3.297 & 0.7 & 0.97 & 0.624 & 1.92 & 9 \\
2 & ADHHR.167 & 3.249 & 1.504 & 3.249 & 0.67 & 0.969 & 0.606 & 1.922 & 9 \\
3 & ADHHR.168 & 3.124 & 1.284 & 3.124 & 0.67 & 0.972 & 0.477 & 1.923 & 9 \\
4 & ADHHR.20 & 3.261 & 1.878 & 3.261 & 0.68 & 0.975 & 0.605 & 1.903 & 9 \\
\hline
\end{tabular}

The top hypotheses showed the survival score of 3.297. The distances and angles between the different pharmacophore features of hypothesis ADHHR.27 were computed and given in Table S5 \& S6 respectively. The pictorial representation of distances between various features is given in figure 9. The pharmacophore hypotheses also yielded a 3D-QSAR model with good PLS statistics (Table 5). In the table, the standard deviation of the regression is the RMS error in the fitted activity values. $\mathrm{R}^{2}$ is the coefficient of regression. A value of 0.80 , means that the model accounts for $80 \%$ of the variance in the observed activity data. R2 is always between 0 and 1 . Stability of the model predictions to changes in the training set composition. Maximum value is 1 . F value is the ratio of the model variance to the observed activity variance. Large values of F indicate a more statistically significant regression. $\mathrm{P}$ is the significance level of $\mathrm{F}$ when treated as a ratio of Chi-squared distributions. Smaller values indicate greater degree of confidence. A P value of 0.05 means $\mathrm{F}$ is significant at the $95 \%$ level. RMSE is the root-mean-square error in the test set predictions. $\mathrm{Q}^{2}$ refers for the predicted activities. It is directly analogous to R-squared, but based on the test set predictions and the Pearson-r value is for the correlation between the predicted and observed activity for the test set. The basic pharmacophore was mapped over the molecule of the dataset and represented in fig. 10(a). The graph was plotted between the predicted activity and actual activity values and showed a correlation $r^{2}=0.9322$ (Figure 10 (b) The fitness scores of training and test set molecules are presented in the Table S7 which shows the fitness of molecules onto the generated hypothesis. 
Table 5. Statistical results of the generated 3D-QSAR models

\begin{tabular}{lllllllll}
\hline PLS & SD & $\mathrm{R}^{2}$ & $\mathrm{~F}$ & $\mathrm{p}$-value & Stability & RMSE & $\mathrm{Q}^{2}$ & Pearson-R \\
\hline 1 & 0.8095 & 0.6114 & 40.9 & $8.943 \mathrm{e}-07$ & 0.8357 & 0.4338 & 0.473 & 0.8993 \\
2 & 0.572 & 0.8135 & 54.5 & $7.671 \mathrm{e}-10$ & 0.4444 & 0.5156 & 0.2556 & 0.7004 \\
3 & 0.2553 & 0.9643 & 216.3 & $1.68 \mathrm{e}-17$ & 0.1392 & 0.5052 & 0.2852 & 0.6884 \\
4 & 0.1744 & 0.984 & 354.6 & $2.656 \mathrm{e}-20$ & 0.137 & 0.4587 & 0.4108 & 0.7373 \\
5 & 0.1264 & 0.992 & 544.2 & $2.815 \mathrm{e}-22$ & 0.182 & 0.503 & 0.2915 & 0.6887 \\
\hline
\end{tabular}

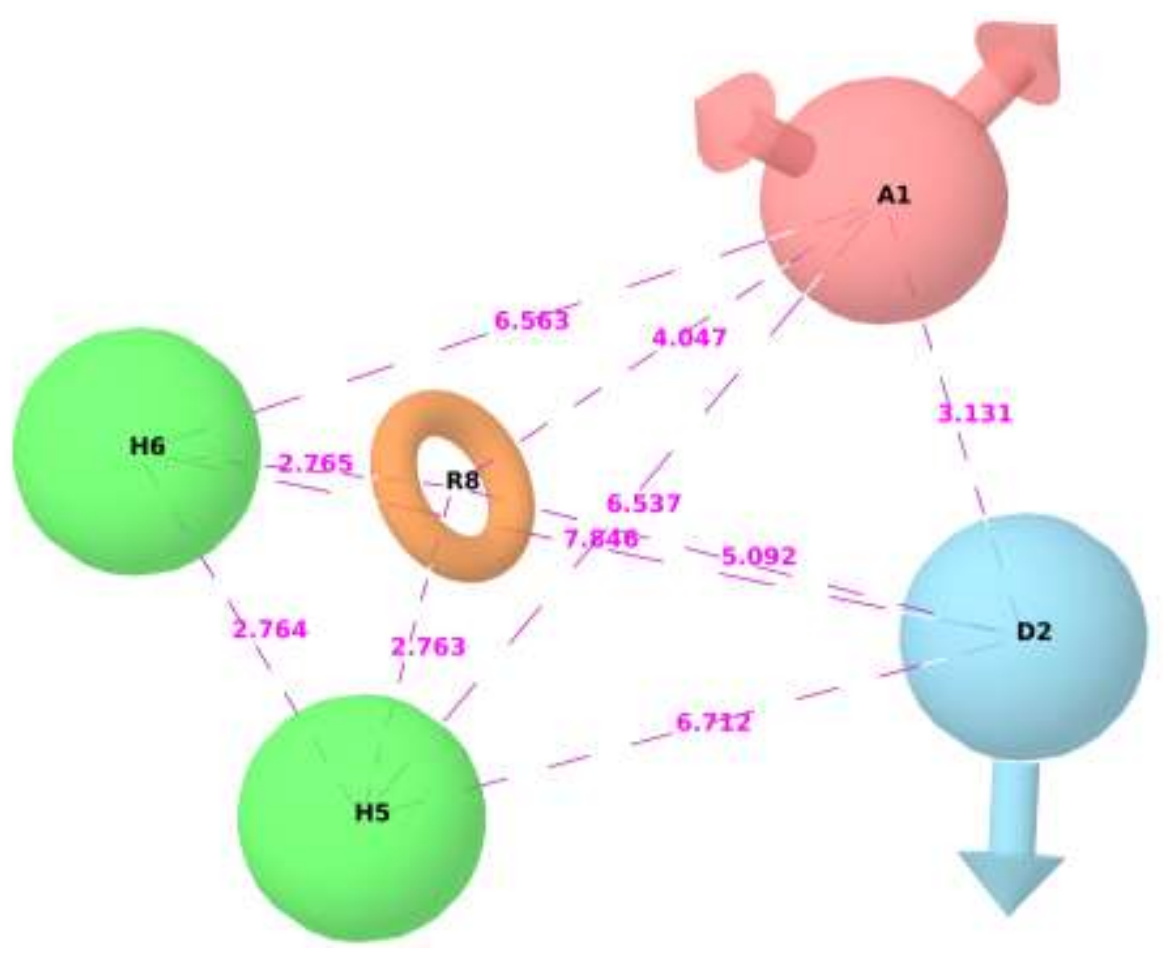

Fig.9. Hypotheses: ADHHR.27, showing distance between different mapped features 


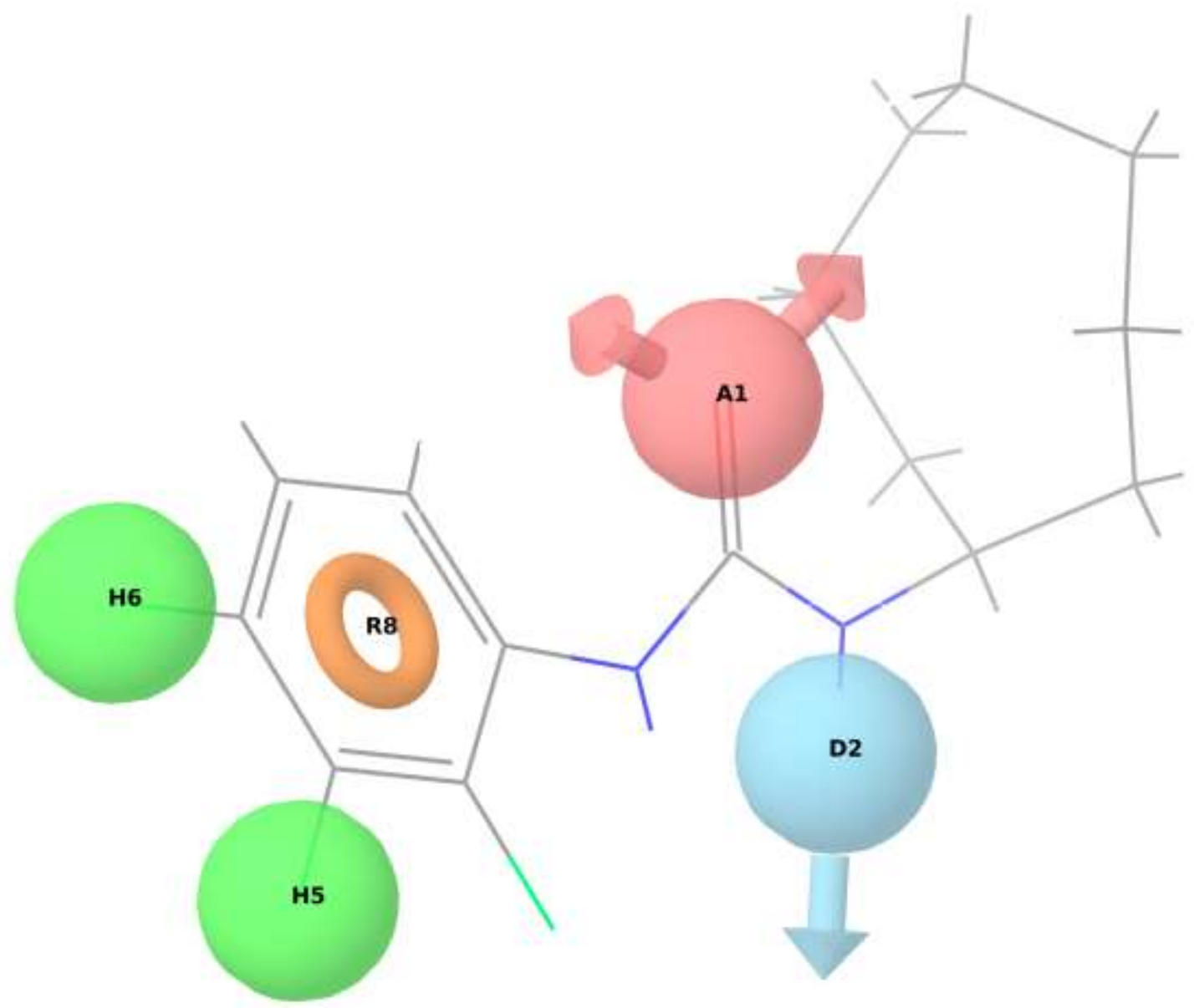

Fig. 10(a). Pharmacophore mapped over the molecule of the dataset

\section{Correlation plot}

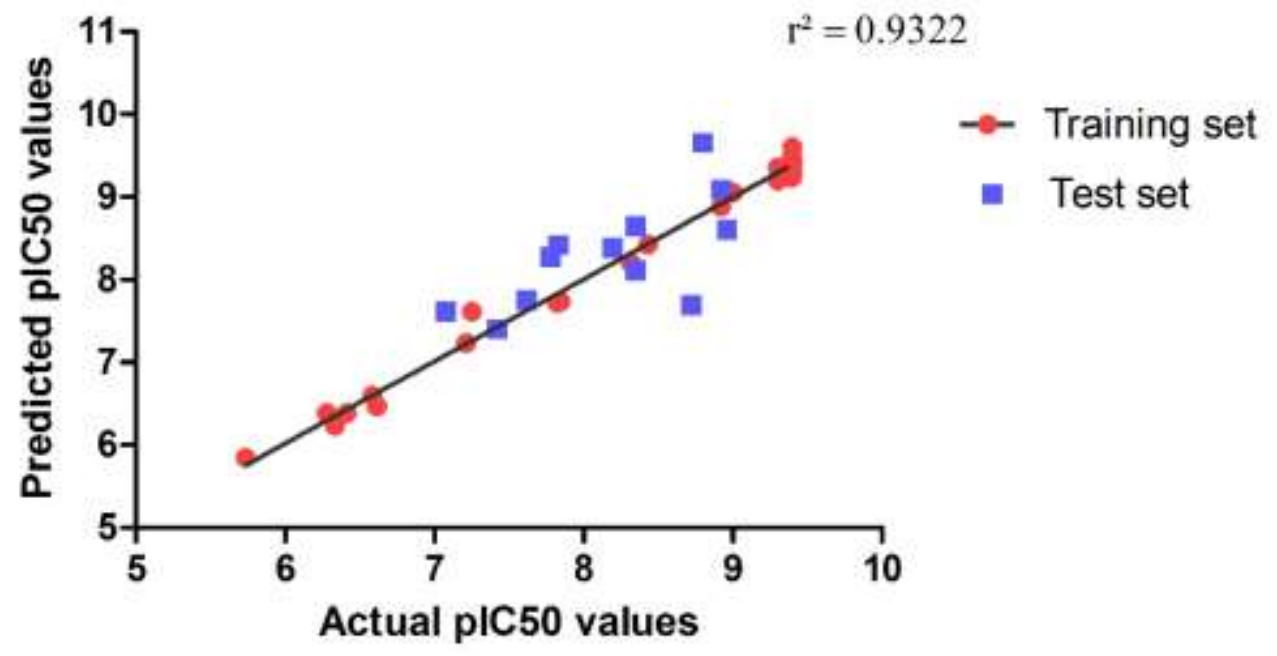

Fig.10(b). Correlation plot between actual and predicted activity values 


\subsubsection{Contour analysis}

The contour analysis is done to find out the blue and red regions present in the pharmacophore. The red regions are the called as contours where substitutions cause depreciation of activity and blue region substitution cause appreciation in activity. These contour plots generated as a result of 3DQSAR model development are displayed as positive and negative activity coefficients of the properties detected as a result of the output of best generated hypotheses (Figure 11). The blue regions represent that the substitution at the positions is favourable for the activity and the red regions represent the devaluating activity. That means, if there is a substitution around blue regions it will be enhancing the activity and if there is substitution around red regions, it will lead to diminish the activity.

\subsubsection{Hydrogen bond acceptor property contour}

The blue contours at the oxygen of urea signified the importance of H-bond acceptor group at this position. (Figure 11 (a)). The H-bond acceptor oxygen is important for anti-tubercular action.

\subsubsection{Hydrogen bond donor property contour}

As shown in figure 11 (b), the blue contours at the nitrogen atom of urea moiety demonstrated the importance of H-bond donor group at this position. This H-bond donor group is important for enzyme inhibition for the treatment of tuberculosis. 


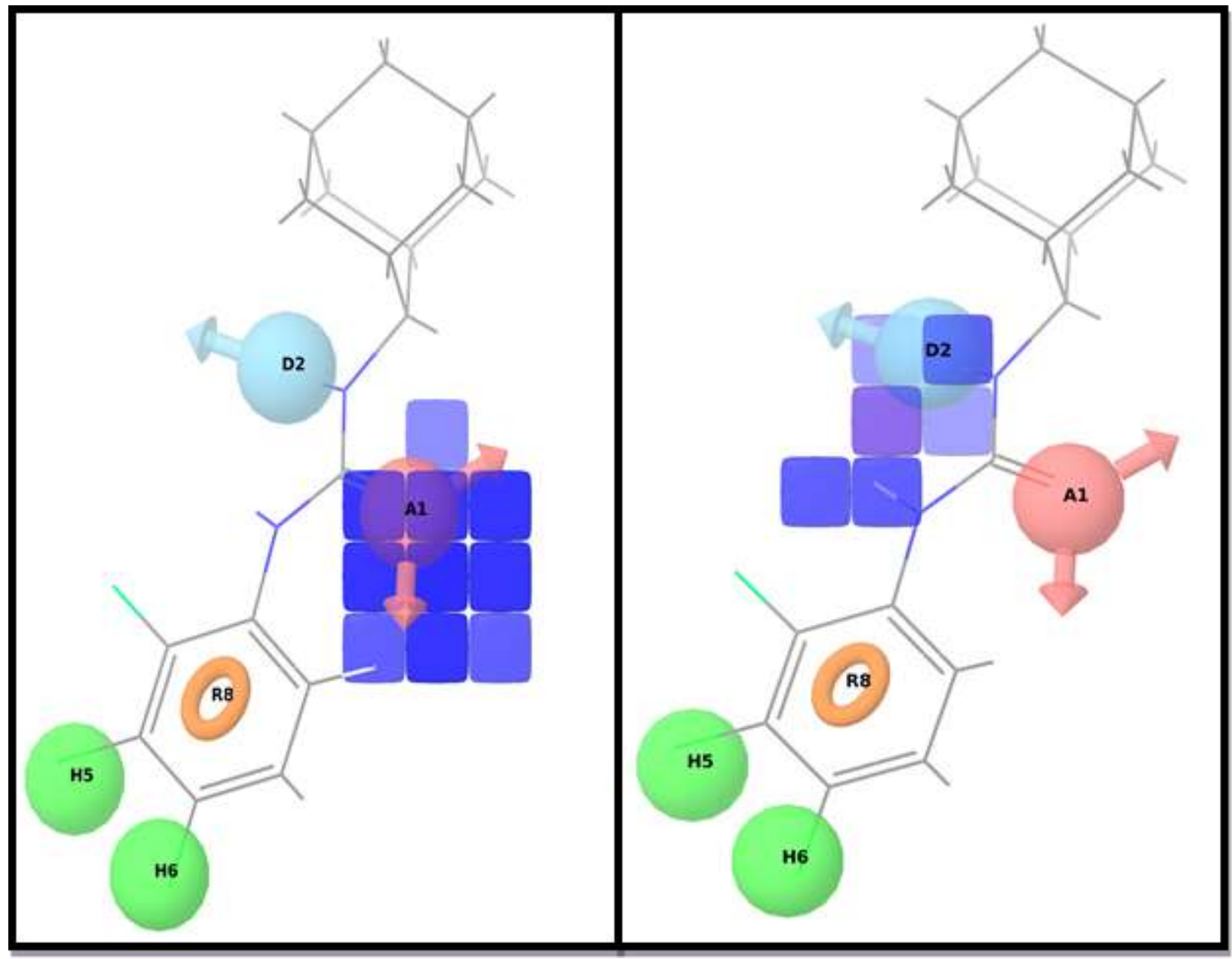

Fig.11. Stereoviews of contour maps for 3D-QSAR model hydrogen bond acceptor (a), hydrogen bond donor (b) properties

The SAR was developed on the basis of the above studies (Figure 12) which demonstrated that the one hydrogen bond acceptor group i.e carbonyl and one hydrogen bond donor i.e. NH played a significant role in the inhibition of the Mtb epoxide hydrolase. The compounds with admantane ring on one side of urea moiety and tri-fluorophenyl ring on other side displayed significant goldscores. The polar groups when substituted at R2 position showed less affinity towards receptor binding and lowered the fitness scores when compared to other derivatives. 


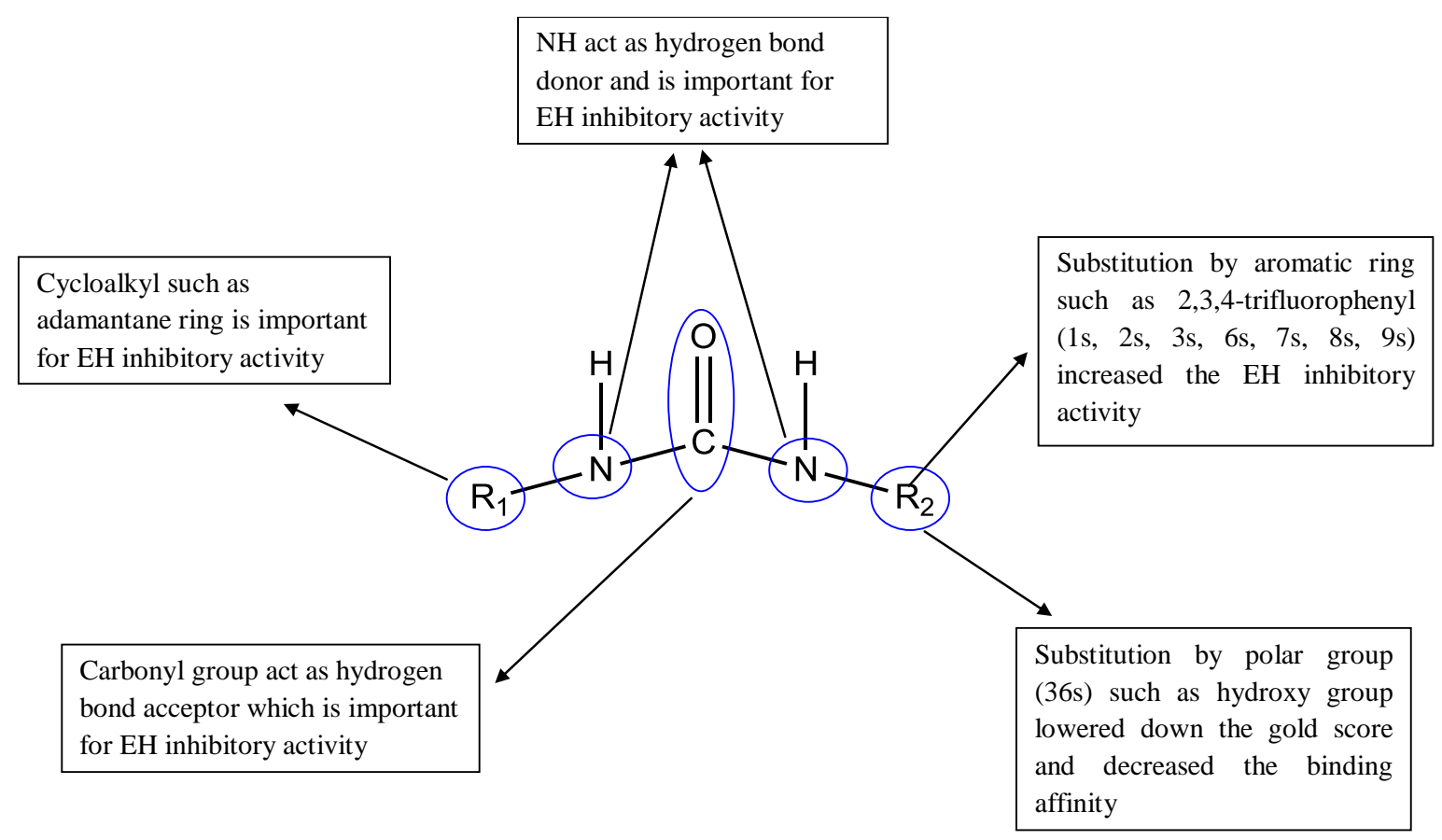

Fig.12. Proposed pictorial representation of structure activity relationship (SAR) of 1,3-disubstituted urea derivatives towards Mtb epoxide hydrolase (EH)

\subsection{Molecular docking analysis}

In pharmacophore mapping part of our study, we found the pharmacophoric features which are essential for the biological activity. This is followed by the next part of our study in which we docked the compounds containing these pharmacophoric features into the binding pocket of epoxide hydrolase which predict their binding orientation at the active sites of epoxide hydrolase. The GOLD, a genetic algorithm based program successfully docked the urea derivative compounds into the active sites of epoxide hydrolase and the most stable conformation based on the CHEMPLP scoring function was considered to analyze various drug receptor interactions. The gold fitness scores of the docked compounds and their $\mathrm{H}$ bond (HB) interactions are shown in Table 6 . 
Table 6. Gold fitness score of 1,3-disubstituted urea analogues and HB formed with amino acid residues of Mtb epoxide hydrolase

\begin{tabular}{|c|c|c|c|c|c|}
\hline Compounds & $\begin{array}{l}\text { Gold fitness } \\
\text { score }\end{array}$ & $\begin{array}{l}\text { Residue involved in } \mathrm{H} \text { Bond } \\
\text { interaction with epoxide } \\
\text { hydrolase }\end{array}$ & Compounds & $\begin{array}{l}\text { Gold } \\
\text { fitness } \\
\text { score }\end{array}$ & $\begin{array}{l}\text { Residue involved in } \mathrm{H} \\
\text { Bond interaction with } \\
\text { epoxide hydrolase }\end{array}$ \\
\hline $1 \mathrm{~s}$ & 88.64 & Asp104 \& Tyr164 & $21 \mathrm{~s}$ & 85.57 & Asp104 \& Tyr164 \\
\hline $2 \mathrm{~s}$ & 98.85 & Asp104 \& Tyr272 & $22 \mathrm{~s}$ & 93.42 & Asp104 \& Tyr164 \\
\hline $3 \mathrm{~s}$ & 96.91 & Asp104 \& Tyr164 & $23 \mathrm{~s}$ & 86.76 & Asp104 \& Tyr164 \\
\hline $4 \mathrm{~s}$ & 89.81 & Asp104 \& Tyr164 & $24 \mathrm{~s}$ & 77.63 & Asp104 \& Tyr164 \\
\hline $5 \mathrm{~s}$ & 93.63 & Asp104 \& Tyr164 & $25 \mathrm{~s}$ & 84.10 & Asp104 \& Tyr164 \\
\hline $6 s$ & 91.19 & Asp104 \& Tyr164 & $26 s$ & 81.82 & Asp104 \& Tyr164 \\
\hline $7 \mathrm{~s}$ & 93.27 & Asp104 \& Tyr164 & $27 \mathrm{~s}$ & 78.06 & Asp104 \& Tyr164 \\
\hline $8 \mathrm{~s}$ & 90.45 & Asp104, Tyr164 \& Tyr272 & $28 \mathrm{~s}$ & 72.77 & Asp104 \& Tyr164 \\
\hline $9 \mathrm{~s}$ & 88.34 & Asp104, Tyr164 \& Tyr272 & $29 \mathrm{~s}$ & 78.06 & Asp104 \& Tyr164 \\
\hline $10 \mathrm{~s}$ & 77.66 & Asp104 & $30 \mathrm{~s}$ & 77.45 & Asp104 \& Tyr164 \\
\hline $11 \mathrm{~s}$ & 88.15 & Tyr272 & $31 \mathrm{~s}$ & 89.23 & \\
\hline $12 \mathrm{~s}$ & 95.05 & Asp104 \& Tyr164 & $32 \mathrm{~s}$ & 89.36 & Asp104 \& Tyr164 \\
\hline $13 \mathrm{~s}$ & 89.43 & Asp104, Tyr164 \& Tyr272 & $33 \mathrm{~s}$ & 87.61 & Tyr164 \& Tyr272 \\
\hline $14 \mathrm{~s}$ & 88.07 & Asp104 \& Tyr164 & $34 \mathrm{~s}$ & 87.74 & Tyr272 \\
\hline $15 \mathrm{~s}$ & 67.23 & Asp104 \& Tyr164 & $35 \mathrm{~s}$ & 82.61 & $\begin{array}{l}\text { Asp104, Tyr164\& } \\
\text { Tyr272 }\end{array}$ \\
\hline $16 \mathrm{~s}$ & 63.19 & Asp104 \& Tyr164 & $36 \mathrm{~s}$ & 71.50 & Asp104 \& Tyr164 \\
\hline $17 \mathrm{~s}$ & 72.61 & Asp104 \& Tyr164 & $37 \mathrm{~s}$ & 79.49 & Asp104 \& Tyr164 \\
\hline $18 \mathrm{~s}$ & 89.30 & Asp104 \& Tyr164 & $38 \mathrm{~s}$ & 81.29 & $\begin{array}{l}\text { Asp104, Tyr164\& } \\
\text { Tyr272 }\end{array}$ \\
\hline $19 \mathrm{~s}$ & 84.21 & Asp104 \& Tyr164 & $39 \mathrm{~s}$ & 79.35 & $\begin{array}{l}\text { Asp104, Tyr164 \& } \\
\text { Tyr272 }\end{array}$ \\
\hline $20 \mathrm{~s}$ & 85.07 & Asp104 \& Tyr164 & $40 \mathrm{~s}$ & 85.54 & $\begin{array}{l}\text { Asp104, Tyr164 \& } \\
\text { Tyr272 }\end{array}$ \\
\hline $\begin{array}{l}\text { Co-crystal } \\
\text { ligand }\end{array}$ & 82.44 & Asp104 \& Tyr164 & & & \\
\hline
\end{tabular}

Overall, these compounds showed good fitness scores which suggested favorable binding of the compounds with that of the enzyme. In the present series, the compound $2 \mathrm{~s}, 3 \mathrm{~s}, \mathbf{5 s}, \mathbf{1 2 s}$ and $22 \mathrm{~s}$ 
showed greater fitness scores as compared to rest of the compounds. The compound $2 \mathrm{~s}$ (containing trifluorophenyl and admantane ring) was found to dock into the active site of the enzyme with a highest fitness score of 98.85. It formed three hydrogen bonds, one with Tyr272 and two with Asp 104; wherein the oxygen atom of the carbonyl group showed $\mathrm{H}$ bond interaction with phenolic oxygen of Tyr272 and two nitrogen atoms of urea showed $\mathrm{H}$ bond interaction with acidic oxygen of Asp104. The trifluorophenyl and 1-adamantyl ring of the 2s is surrounded by Phe36, Pro37, Asp104, Trp105, Ile137, Leu226, Tyr272 and Trp307 amino acid residues (figure 13). The interaction with the Tyr272, Tyr164 and Asp104 is important for the enzyme inhibitory activity. Moreover, to validate the process, co-crystallized ligand (1,3-diphenyl urea/carbanilide) was extracted and redocked into the active site of the enzyme. The conformation of docked ligand was found similar to the conformation of co-crystal ligand. The comparison of docked and crystal structure conformation is shown in figure 14(a). It was found to show the H bond interaction with the Tyr164 and Asp104 (figure 14 (b)) as similar to the majority of compounds and displayed a goldscore of 82.44; wherein the carbonyl oxygen interacted with the oxygen of the phenolic group of Tyr164 and two nitrogens interacted with the acidic oxygen of Asp 104. This carbanilide based inhibitor represents an outstanding scaffold to design and develop potential structural based inhibitors against the Mtb EH.

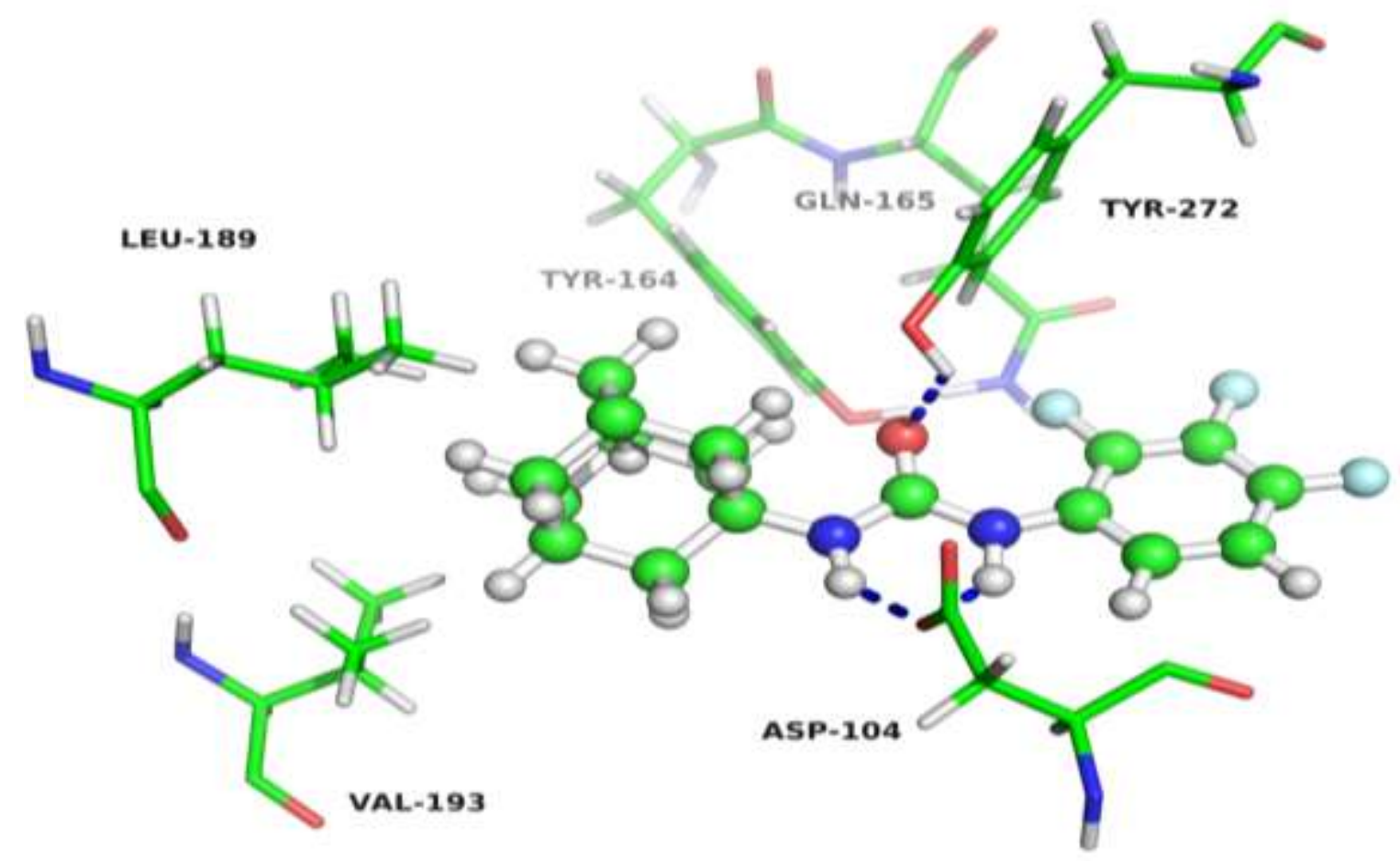

Fig.13. Docking interaction pose of compound 2s at the active site of Mtb epoxide hydrolase; interacting residues are shown as sticks whereas compound $2 \mathrm{~s}$ is shown in ball and stick representation 


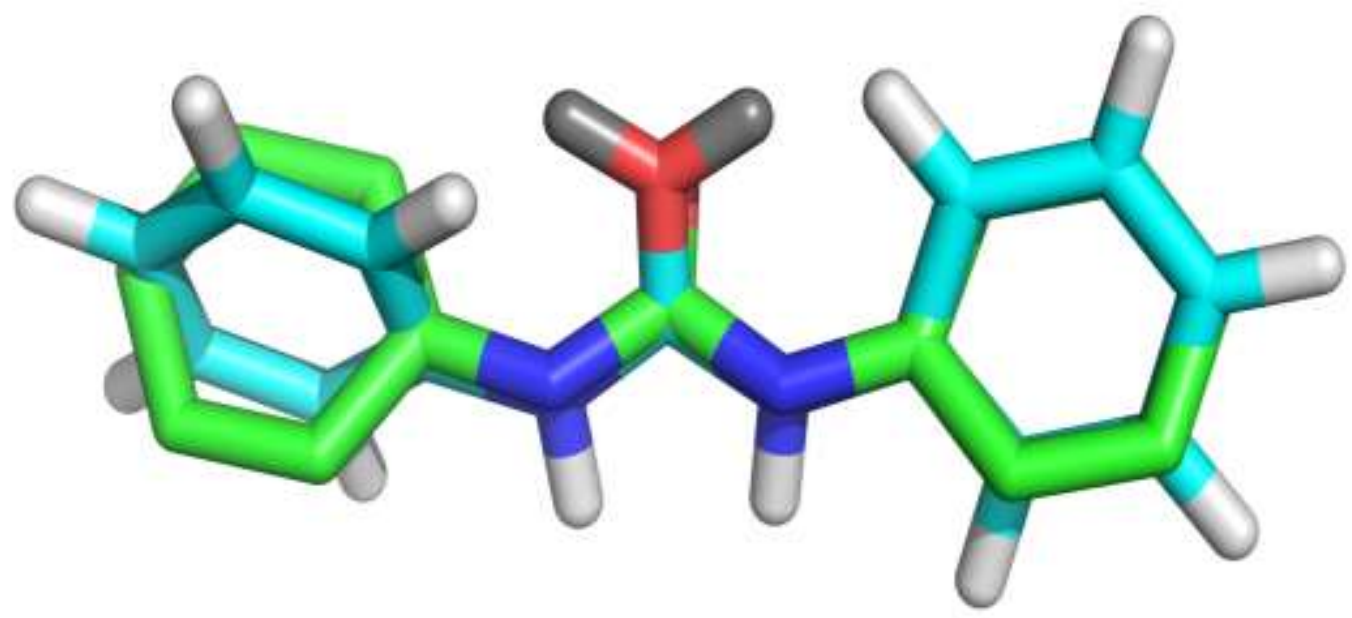

Fig. 14(a). Superimposition of co-crystal ligand over the docked conformation of the same ligand

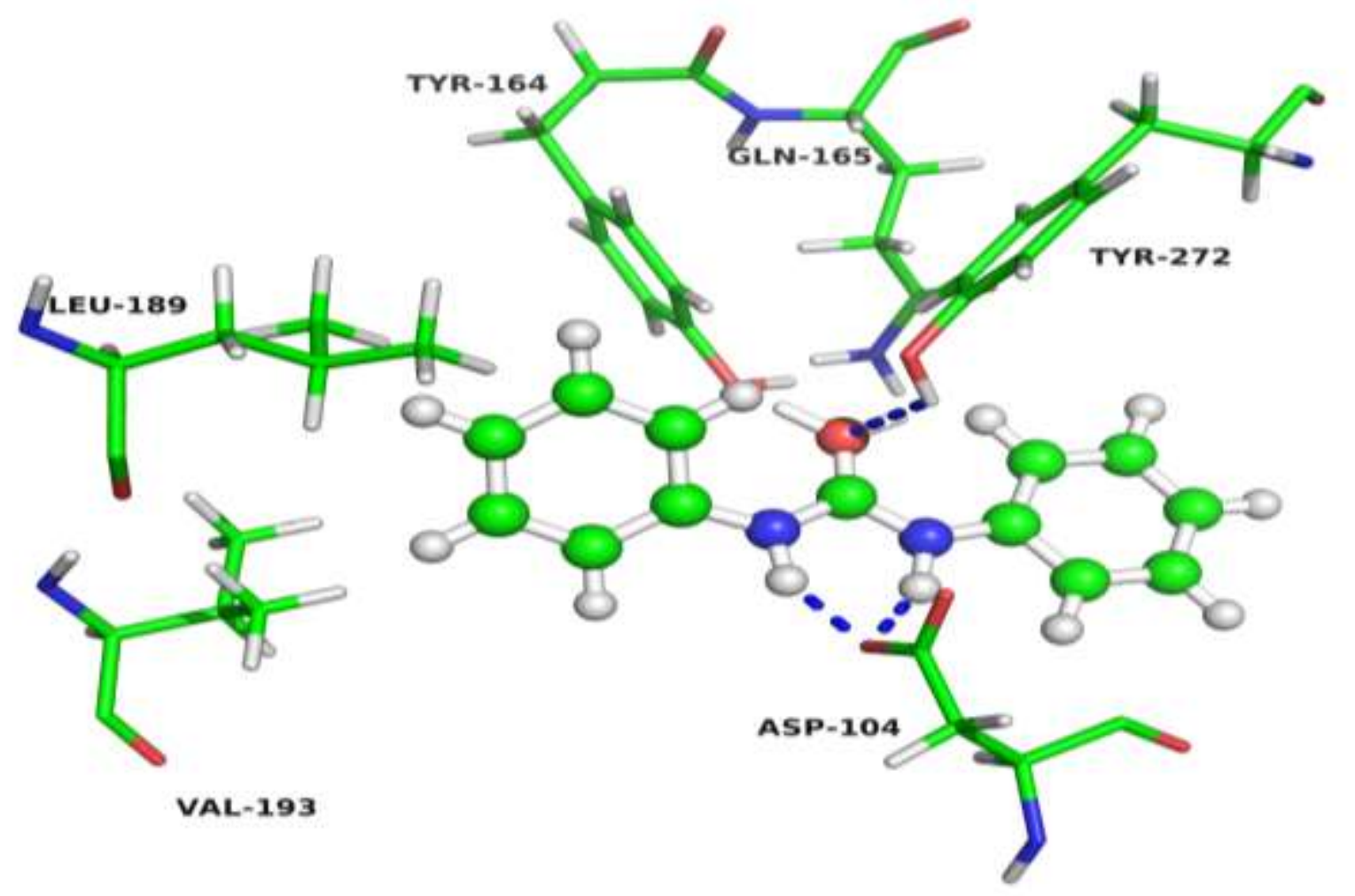

Fig.14(b). Docking interaction pose of 1,3-diphenyl urea (co-crystallized ligand) at the active site of Mtb epoxide hydrolase; interacting residues are shown as sticks whereas co-crystal ligand is shown in ball and stick representation

\subsection{Molecular dynamics simulations}

The docked complex systems were prepared using molecular docking protocol mentioned in the previous section. These docked binary complexes comp_2s, comp_15s and comp_37s i.e. epoxide 
hydrolase (PDB ID: 2ZJF) in complex with molecules 2s, 15s and 37s were considered for molecular dynamics (MD) simulations studies.

\subsubsection{RMSD and B-factor analysis}

To estimate the stability of the trajectories, RMSD calculation was carried out (Figure 15 (a)). The trajectories showed that RMSD gets stabilized after 6 ns of simulation. The comp_2s showed an average RMSD of $1.79 \AA$ with standard deviation (SD) of \pm 0.33 . Similarly, comp_37s showed an average RMSD of $1.98 \AA$ with SD \pm 0.24 . The comp_15s showed lowest average RMSD of $1.52 \AA$ with $\mathrm{SD} \pm 0.19$. All complexes showed overall as well as average RMSD below $2 \AA$ throughout the 10 ns simulations which showed MD trajectories are stable and can be used for further analysis to calculate the B-factor, H-bond and distance analysis.

The estimation of the B-factor showed the flexibility of various parts of epoxide hydrolase enzyme (Figure 15 (b)). The N-terminal of the epoxide hydrolase showed a B-factor of about $\sim 100$ $\AA^{2}$ while C-terminals showed a maximum B-factor of about $\sim 1700 \AA^{2}$. It indicated that C-terminal is highly flexible part among all the domains available in the epoxide hydrolase enzyme. In addition to $\mathrm{C}$-terminal, residue ranging $\sim 200$ to 220 as well as residue around $\sim 150$ showed a B-factor value $\geq 100 \AA^{2}$ indicating second most flexible part in the epoxide hydrolase enzyme. The flexibility of this part is in agreement of expectation as it covers active site cavity where epoxide hydrolase inhibitors bind.

(A) RMSD

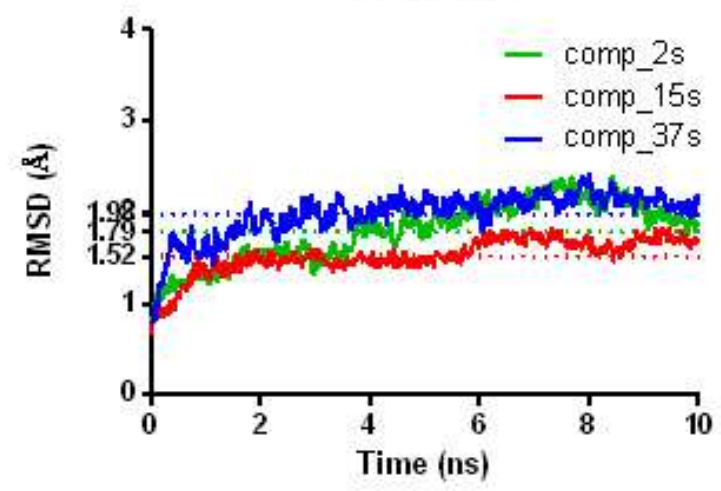

(B) B-factor

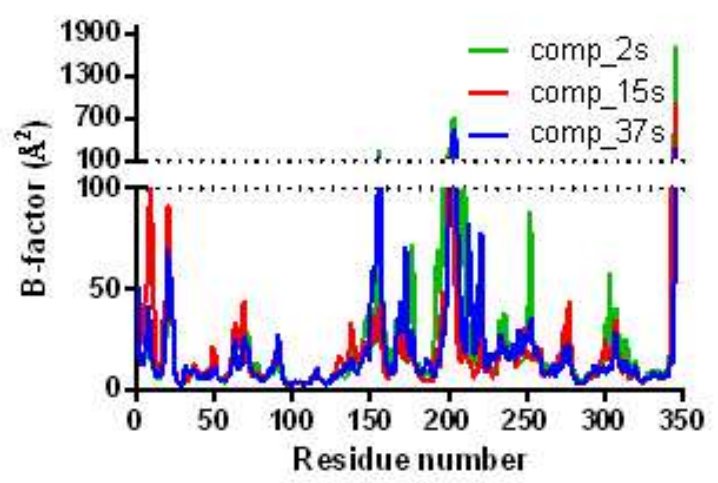

Fig.15 (a) RMSD and (b) B-factor calculations for the MD trajectory. 


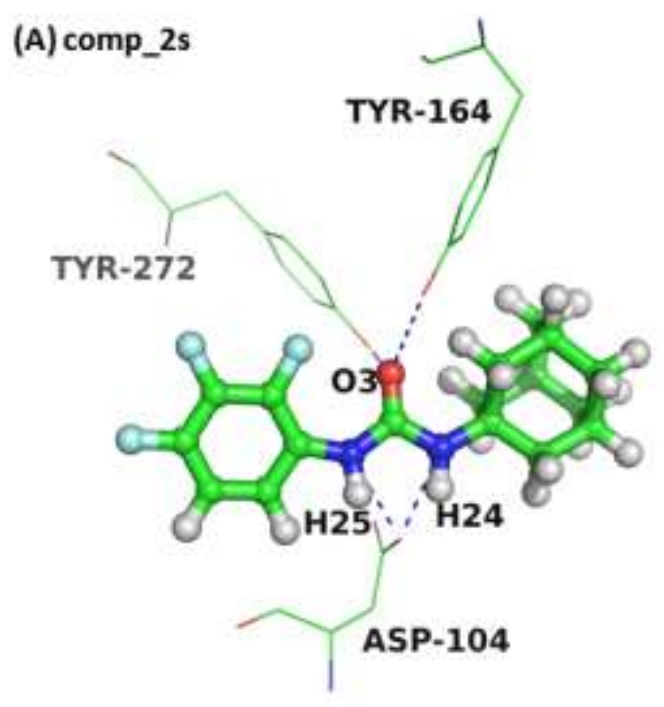

(B) comp_15s
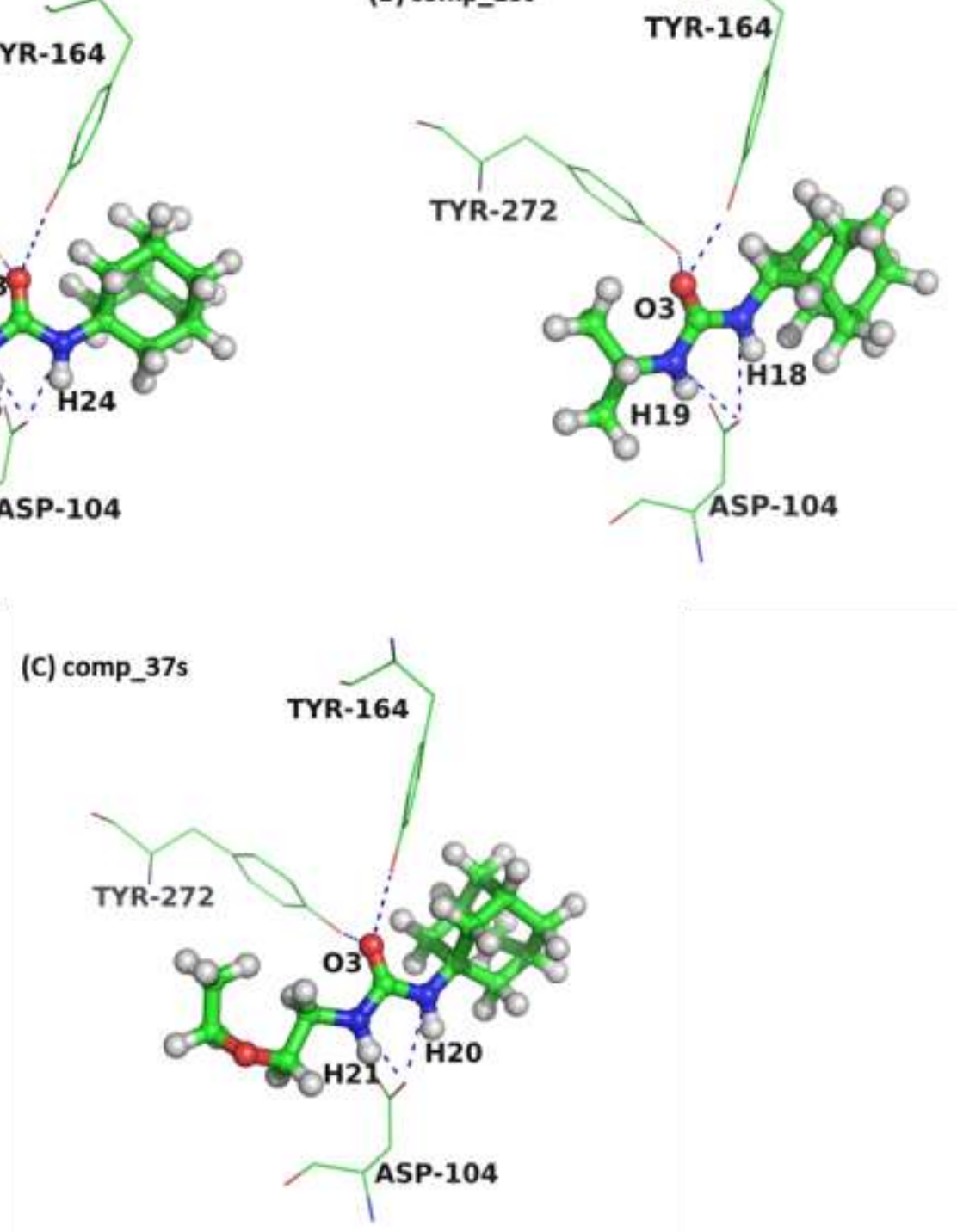

Fig. 16. Interaction of epoxide hydrolase with molecule (a) $2 \mathrm{~s}$, (b) $15 \mathrm{~s}$ and (c) $37 \mathrm{~s}$ in the analyzed complexes comp_2s, comp_15s and comp_37s respectively

\subsubsection{Epoxide hydrolase-inhibitor interaction analysis}

The B-factor analysis showed that active site residues of epoxide hydrolase are flexible enough to interact with the bound inhibitor. The analysis of protein-ligand complex is very important to emphasize on pre-existing non-bonded interactions [63, 64]. Also, it provides an opportunity to identify hotspot residues involved in protein-ligand interactions [65-70]. The complexes comp_2s, comp_15s and comp_37s were analyzed for the protein-ligand interactions to identify the active site residues involved in the non-bonded interactions. The residues within $6 \AA$ around the bound 
inhibitors were considered as active site residues and analyzed for the pre-existing interactions. Only, three residues Asp104, Tyr164 and Tyr272 were observed to make the H-bond interactions with the bound ligand 2s, 15s and 37s (Figure 16). In complex comp_2s, COO ${ }^{-}$group of Asp104 was observed to make H-bond interactions with $\mathrm{H} 24$ and $\mathrm{H} 25$ atoms of amino groups in molecule 2s with H-bond occupancy of $67.67 \%$ (Table 7). In addition, OH group of Tyr164 and Tyr272 was observed to make H-bond interactions with $\mathrm{O} 3$ atom of carboxyl with $\mathrm{H}$-bond occupancy $54.50 \%$ and $61.30 \%$ respectively.

Similarly, in complex comp_15s, $\mathrm{COO}^{-}$group of Asp104 was found to form H-bond interactions with $\mathrm{H} 18$ and $\mathrm{H} 19$ atoms of amino groups in molecule 15s with H-bond occupancy $37.30 \%$ while $\mathrm{OH}$ group of Tyr164 and Tyr272 was observed to make H-bond interactions with O3 atom of carboxyl group with H-bond occupancy $75.90 \%$ and $66.10 \%$ respectively. In the complex comp_37s, $\mathrm{COO}^{-}$group of Asp104 was observed to make H-bond interactions with H20 and $\mathrm{H} 21$ atoms of amino groups in molecule 37s with H-bond occupancy $48.10 \%$. And, OH group of Tyr164 and Tyr272 was observed to make H-bond interactions with $\mathrm{O} 3$ atom of carboxyl group with H-bond occupancy $77.60 \%$ and $69.20 \%$ respectively.

Table 7. H-bond occupancy with molecules 2 s, 15 s and $37 \mathrm{~s}$

\begin{tabular}{|c|c|c|}
\hline \multicolumn{3}{|c|}{ H-bond occupancy with molecule 2 s in comp_2s } \\
\hline S.No & Residue & H-bond occupancy \\
\hline 1. & Asp104 & $67.67 \%$ \\
\hline 2. & Tyr164 & $54.40 \%$ \\
\hline 3. & Tyr272 & $61.30 \%$ \\
\hline \multicolumn{3}{|c|}{ H-bond occupancy with molecule $15 \mathrm{~s}$ in comp_15s } \\
\hline 1. & Asp104 & $37.30 \%$ \\
\hline 2. & Tyr164 & $75.90 \%$ \\
\hline 3. & Tyr272 & $66.10 \%$ \\
\hline \multicolumn{3}{|c|}{ H-bond occupancy with molecule 37s in comp_37s } \\
\hline 1. & Asp104 & $48.10 \%$ \\
\hline 2. & Tyr164 & $77.60 \%$ \\
\hline 3. & Tyr272 & $69.20 \%$ \\
\hline
\end{tabular}


(A) Comp_2s

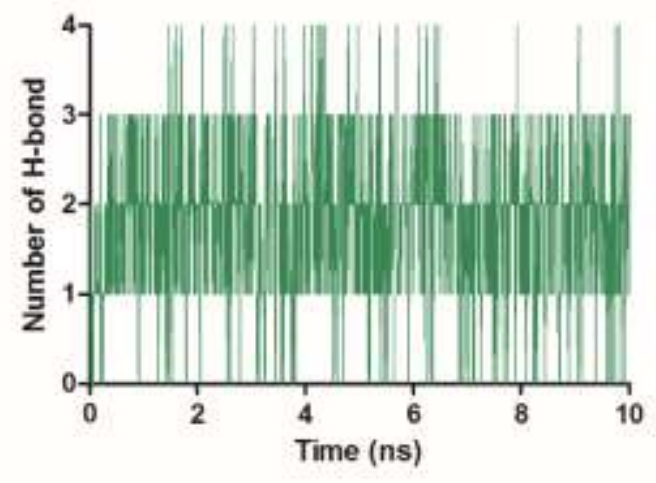

(B) Comp_15s

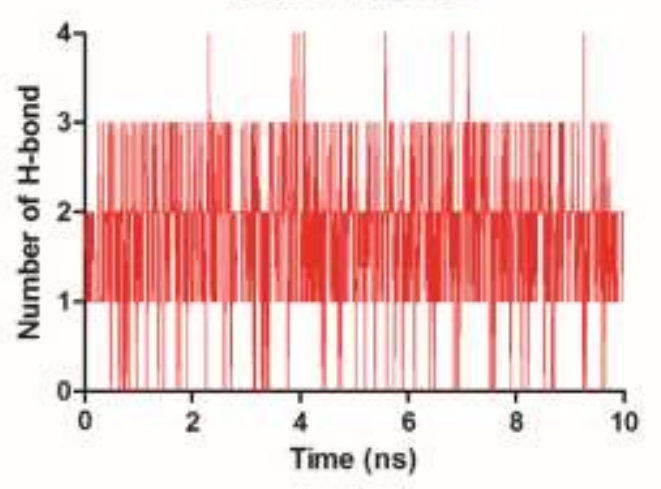

(C) Comp_37s

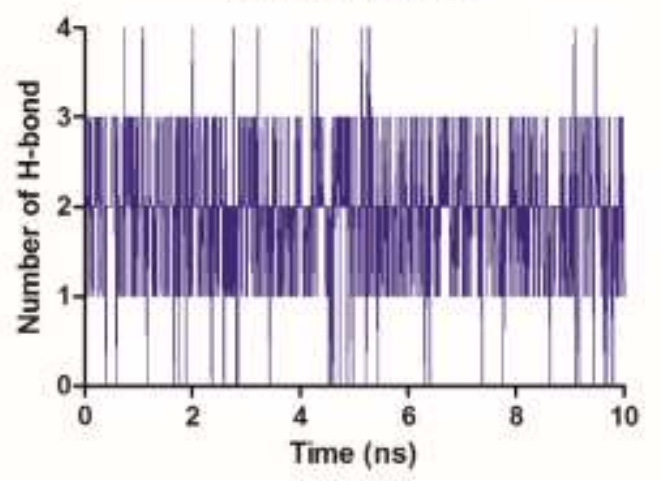

(a) Comp_2s

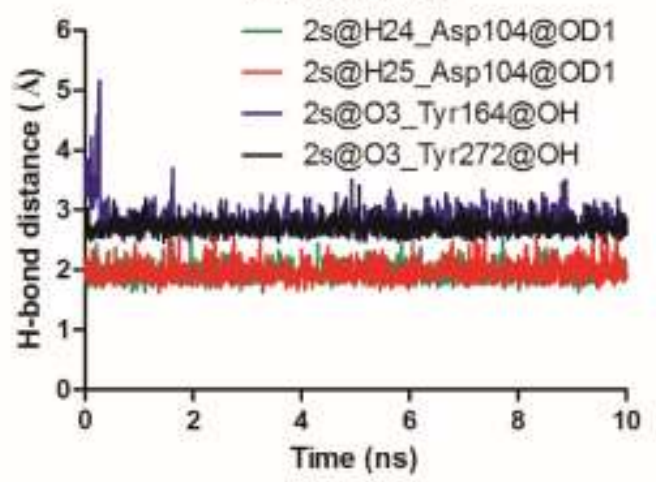

(b) Comp_15s

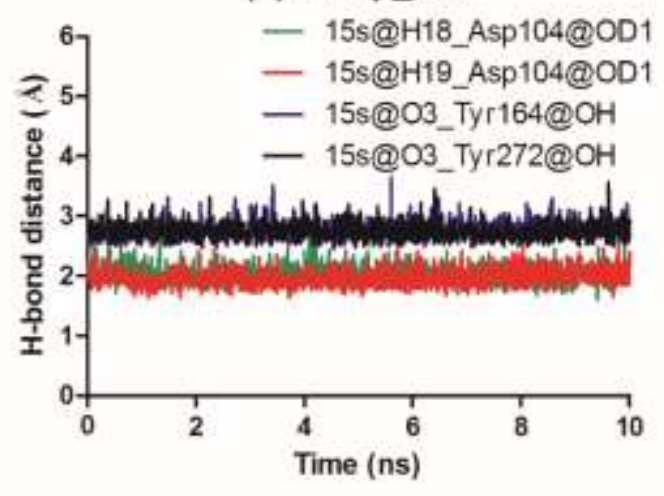

(c) Comp_37s

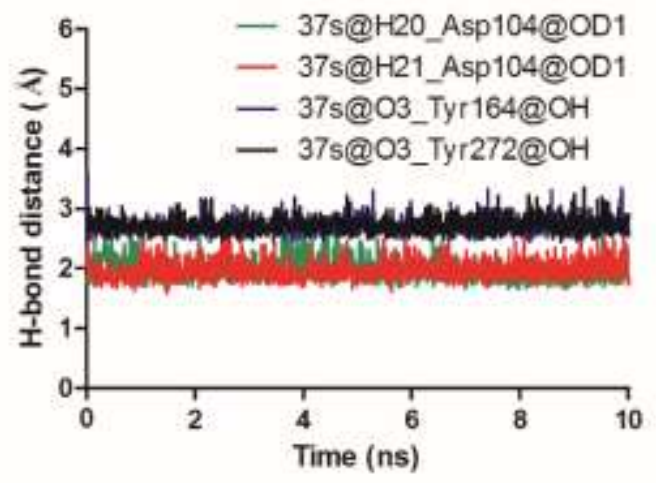

Fig.17. Number of $\mathbf{H}$-bond interactions and $\mathbf{H}$-bond distance in the complexes comp_2s, comp_15s and comp_37s. 
The average H-bond interaction was observed 1.87 between all binding residues and molecule $2 \mathrm{~s}$ while average binding interaction of about 1.79 was observed between all binding residues and molecule 15s (Figure 17 (A) and 17 (B)). The highest average H-bond interaction of about 1.94 was observed between all binding residues and molecule 37s (Figure 17 (C)). It is very little opposite to the obtained docking score where molecule $2 \mathrm{~s}$ was observed with highest molecule docking score of 98.85 and molecule 37s was observed with moderate docking score of about 79.49. However, similar to average H-bond interaction observation, molecule 15s was found with lowest docking score of 67.23. This observation of average H-bond interaction is in agreement with the obtained H-bond occupancy results where molecule 37s was observed to have highest H-bond occupancy with binding site residues. The H-bond distances were observed between the lengths ranging from $1.8 \AA$ to $2.8 \AA$ (Figure 17 (a), 17 (b) and 17 (c)).

\subsubsection{MM-GBSA binding free energy analysis}

The MM-GBSA binding free energy for the molecule $2 \mathrm{~s}, \mathbf{1 5 s}$ and $37 \mathrm{~s}$ was estimated to verify the observed H-bond interaction contributions. Similar to the observed average H-bond analysis, the value of $\Delta \mathrm{G}_{\mathrm{total}}=-52.24 \mathrm{kcal} / \mathrm{mol}$, was observed highest for the molecule 37s. It was found $\Delta \mathrm{G}_{\text {total }}$ $=-51.70 \mathrm{kcal} / \mathrm{mol}$ and $\Delta \mathrm{G}_{\mathrm{total}}=-49.97 \mathrm{kcal} / \mathrm{mol}$ for the molecules $2 \mathrm{~s}$ and $15 \mathrm{~s}$ respectively. The van der Waals interactions showed slightly poor contribution towards ligand binding as compared to electrostatic interactions in case of comp_2s and comp_37s while both interactions found almost

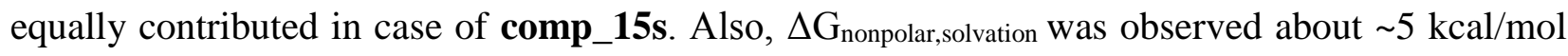
which was similar in cases of all three complexes (Table 8) Further, the observed binding free energy is in agreement of the H-bond interactions between epoxide hydrolase and inhibitors. The $\Delta \mathrm{G}$ was contributed by the various binding site residues, thus, decomposition of free energy into per-residue can help to identify and estimate role of particular residue in the ligand binding.

Table 8. Binding free energy components estimated for the complexes ( $\mathrm{kcal} / \mathrm{mol})$

\begin{tabular}{llll}
\hline Energy components & Comp_2s & Comp_15s & Comp_37s \\
\hline$\Delta \mathrm{E}_{\mathrm{vdW}}$ & -43.33 & -39.06 & -40.36 \\
$\Delta \mathrm{E}_{\text {elec }}$ & -48.87 & -37.94 & -46.75 \\
$\Delta \mathrm{G}_{\text {polar, solvation }}$ & 45.82 & 31.80 & 40.10 \\
$\Delta \mathrm{G}_{\text {nonpolar, solvation }}$ & -5.31 & -4.77 & -5.22 \\
$\Delta \mathrm{G}_{\text {total }}$ & -51.70 & -49.97 & -52.24 \\
\hline
\end{tabular}




\subsubsection{Per-residue free energy decomposition}

To find the function of particular residue in binding of ligand, free energy decomposition was performed. The residues involved in $\mathrm{H}$-bond interactions were observed to contribute differently as Asp104 showed very little $\Delta \mathrm{G}=-0.13 \mathrm{kcal} / \mathrm{mol}$ towards molecule 2s. While Asp104 contributed negatively as $\Delta \mathrm{G}$ was found $+4.29 \mathrm{kcal} / \mathrm{mol}$ and $+2.35 \mathrm{kcal} / \mathrm{mol}$ towards $15 \mathrm{~s}$ and $37 \mathrm{~s}$ respectively. The residues Tyr164 and Tyr272 contributed $\Delta \mathrm{G}$ of about $\sim 1 \mathrm{kcal} / \mathrm{mol}$ towards all molecules. In addition to residues involved in H-bond interactions, free energy decomposition analysis also helped in identification of additional residues involved in non-bonded hydrophobic or van der Waals interactions. Among these residues, Trp105 contributed highest $\Delta \mathrm{G}$ of $\sim 4.19 \mathrm{kcal} / \mathrm{mol},-3.42$ $\mathrm{kcal} / \mathrm{mol}$ and $-3.87 \mathrm{kcal} / \mathrm{mol}$ towards binding of molecules $2 \mathrm{~s}, \mathbf{1 5} \mathrm{s}$ and $37 \mathrm{~s}$ respectively. In addition, residues Ile137, Gln165, Leu189, Val193 and Val296 were identified to make the hydrophobic interactions with the molecules $2 \mathrm{~s}, \mathbf{1 5 s}$ and $37 \mathrm{~s}$ (Table 9).

Table 9. Per-residue decomposition of binding free energy $(\mathrm{kcal} / \mathrm{mol})$

\begin{tabular}{lllll}
\hline S.No. & Residue & Comp_2s & Comp_15s & Comp_37s \\
\hline 1. & Asp104 & -0.13 & 4.29 & 2.35 \\
2. & Trp105 & -4.18 & -3.42 & -3.87 \\
3. & Ile137 & -1.33 & -0.76 & -1.58 \\
4. & Tyr164 & -1.14 & -1.84 & -1.70 \\
5. & Gln165 & -1.18 & -1.46 & -2.04 \\
6. & Leu189 & -0.83 & -1.05 & -1.00 \\
7. & Val193 & -0.75 & -1.04 & -1.01 \\
8. & Tyr272 & -1.00 & -1.01 & -1.06 \\
9. & Val296 & -0.82 & -1.43 & -1.45 \\
\hline
\end{tabular}

\section{CONCLUSION}

The 1,3-disubstituted urea derivatives were subjected to an integrated approach involving QSAR, pharmacophore mapping, molecular docking and molecular dynamics simulation studies. The QSAR studies revealed that the descriptors as SssOE-index, SsssNcount, chi1, T_2_N_5, SdOcount and k2alpha were found to contribute towards the biological activity. When docked, the ligand $2 \mathbf{s}$ containing adamantly ring on one end and 2,3,4-trifluorophenyl on the other end of urea moiety showed a highest gold score of 98.85. The amino acids Asp104, Tyr164 and Tyr272 were found to be involved in $\mathrm{H}$-bonding interaction and played a key role in ligand-receptor binding interactions. 
To validate the docking results, 1,3-diphenylurea was extracted and re-docked into the pocket of epoxide hydrolase and was found to achieve gold score of 82.44 and showed H-bond interaction with Asp104 and Tyr164 amino acid residues. Also, the docked conformation is found almost similar to that of the co-crystal ligand. The molecular dynamics studies of three selected docked complexes showed that the complexes are stable with average RMSD below $2 \AA$. The residues such as Asp104, Tyr164 and Tyr272 made H-bond interactions with bound ligand 2s, 15s and 37s with average H-bond occupancy of $61.12 \%, 59.77 \%$ and $65.0 \%$ respectively. Similar to H-bond analysis, the binding free energy was observed highest for $37 \mathrm{~s}$ and lowest for $15 \mathrm{~s}$. The residues involved in H-bond interactions such as Tyr164 and Tyr272 contributed $\Delta \mathrm{G}$ of about $\sim 1 \mathrm{kcal} / \mathrm{mol}$ towards all molecules. The important structural features that are inferred from our study include that a) The descriptors such as chi1, SdOcount and k2alpha have their role in enhancing the activity and the descriptors such as SssOE-index, SsssNcount and T_2_N_7 play role in decreasing the activity. b) The group as hydrogen bond acceptor, hydrogen bond donor, aromatic ring and hydrophobic group too is required for the epoxide hydrolase inhibitors for exhibiting the anti-TB activity. c) The presence of polar group such as hydroxyl group can lower down the binding affinity and therefore the anti-TB activity. d) The active site amino acids residues involved in H-bond interactions include Asp104, Tyr164 and Tyr272. e) The active amino acid residues are flexible enough to interact with the inhibitors. f) The residues as Trp105, Ile137, Gln165, Leu189, Val193 and Val296 were identified to make the hydrophobic interactions with the molecules. Among these, Trp105 contributed highest as revealed by $\Delta \mathrm{G}$ values. These findings have opened a path for the development of potent inhibitor analogues against $\mathrm{Mtb} \mathrm{EH}$ and provided crucial clues and guidance regarding important structural features that can be used in successful designing of novel highly active analogues against Mtb EH.

\section{ACKNOWLEDGEMENT}

SA wishes to thanks to AICTE and Department of science and Technology, New Delhi for providing financial assistance to carry out this research work. Authors are also thankful to Vinod Deveraji, Applications Scientist, Schrodinger for scientific inputs.

\section{List of Abbreviations}

Mtb-Mycobacterium tuberculosis; TB-Tuberculosis; EH-Epoxide Hydrolase; QSAR-Quantitative Structure Activity Relationships; GOLD-Genetic Optimization for Ligand Docking; MDSMolecular Dynamics Simulation. 


\section{REFERENCES}

1. A. Martínez, S. Torello and R. Kolter, Sliding motility in mycobacteria. Journal of bacteriology. 181 (1999) 7331-7338.

2. L. M. Fu and C. S. Fu-Liu, Is Mycobacterium tuberculosis a closer relative to Gram-positive or Gramnegative bacterial pathogens? Tuberculosis (Edinb). 82 (2002) 85-90.

3. J. E. Galagan, Genomic insights into tuberculosis. Nature Reviews. Genetics. 15 (2014) 307.

4. S. Swaminathan, et al., Recent developments in genomics, bioinformatics and drug discovery to combat emerging drug-resistant tuberculosis. Tuberculosis. 101 (2016) 31-40.

5. J. B. Nachega and R. E. Chaisson, Tuberculosis drug resistance: a global threat. Clinical Infectious Diseases. 36 (2003) S24-S30.

6. Y. Zhang, Persistent and dormant tubercle bacilli and latent tuberculosis. Front Biosci. 9 (2004) 11361156.

7. M. C. Raviglione and A. Pio, Evolution of WHO policies for tuberculosis control, 1948-2001. The Lancet. 359 (2002) 775-780.

8. Y. Zhang, The magic bullets and tuberculosis drug targets. Annu. Rev. Pharmacol. Toxicol. 45 (2005) 529-564.

9. S. Khasnobis, V. E. Escuyer and D. Chatterjee, Emerging therapeutic targets in tuberculosis: postgenomic era. Expert opinion on therapeutic targets. 6 (2002) 21-40.

10. P. S. Jadhavar, et al., Synthesis, biological evaluation and structure-activity relationship of 2styrylquinazolones as anti-tubercular agents. Bioorganic \& medicinal chemistry letters. 26 (2016) 2663 2669.

11. B. Tanwar, et al., Design, development of new synthetic methodology, and biological evaluation of substituted quinolines as new anti-tubercular leads. Bioorganic \& medicinal chemistry letters. 26 (2016) 5960-5966.

12. S. Pancholia, et al., Benzo [d] thiazol-2-yl (piperazin-1-yl) methanones as new anti-mycobacterial chemotypes: Design, synthesis, biological evaluation and 3D-QSAR studies. European Journal of Medicinal Chemistry. 116 (2016) 187-199.

13. P. S Jadhavar, et al., Oxazolidinones as anti-tubercular agents: discovery, development and future perspectives. Current medicinal chemistry. 22 (2015) 4379-4397.

14. G. Sulis, et al., Recent developments in the diagnosis and management of tuberculosis. NPJ primary care respiratory medicine. 26 (2016) 16078.

15. M. D. Segall and C. Barber, Addressing toxicity risk when designing and selecting compounds in early drug discovery. Drug discovery today. 19 (2014) 688-693.

16. F. o. Curtin and P. Schulz, Assessing the benefit: risk ratio of a drug-randomized and naturalistic evidence. Dialogues in clinical neuroscience. 13 (2011) 183.

17. I. R. Edwards and J. K. Aronson, Adverse drug reactions: definitions, diagnosis, and management. The Lancet. 356 (2000) 1255-1259.

18. B. K. Biswal, et al., The molecular structure of epoxide hydrolase B from Mycobacterium tuberculosis and its complex with a urea-based inhibitor. Journal of molecular biology. 381 (2008) 897-912.

19. P. Johansson, et al., Structure of an atypical epoxide hydrolase from Mycobacterium tuberculosis gives insights into its function. Journal of molecular biology. 351 (2005) 1048-1056.

20. C. Morisseau, Role of epoxide hydrolases in lipid metabolism. Biochimie. 95 (2013) 91-95.

21. I. M. Kapetanovic, Computer-aided drug discovery and development (CADDD): in silico-chemicobiological approach. Chemico-biological interactions. 171 (2008) 165-176.

22. G. Sliwoski, et al., Computational methods in drug discovery. Pharmacological reviews. 66 (2014) 334395.

23. T. P. Lybrand, Ligand protein docking and rational drug design. Current opinion in structural biology. 5 (1995) 224-228.

24. N. S. Pagadala, K. Syed and J. Tuszynski, Software for molecular docking: a review. Biophysical reviews. (2017) 1-12. 
25. M. Karplus and J. A. McCammon, Molecular dynamics simulations of biomolecules. Nature Structural \& Molecular Biology. 9 (2002) 646-652.

26. M. P. Allen, Introduction to molecular dynamics simulation. Computational soft matter: from synthetic polymers to proteins. 23 (2004) 1-28.

27. A. K. Jain, et al., QSAR analysis of B-ring-modified diaryl ether derivatives as a InhA inhibitors. Medicinal Chemistry Research. 21 (2012) 145-151.

28. S. Agarwal, et al., QSAR studies involving 2d, 3dqsarand pharmacophore mapping studies on arylsulfonyl imidazolidinone derivatives as anticancer agents. Bulletin of Pharmaceutical Research. 5 (2015) 70-80.

29. S. Agarwal, et al., In-silico pharmacophore mapping and docking studies of indole/benzoximidazole-5carboximidine derivatives as anti-cancer agents. International journal of pharmaceutical sciences and research. 7 (2016) 1000-1019.

30. S. Agarwal, et al., Structure based molecular docking studies on some 1, 3-disubstituted urea derivatives as anti-tubercular agents. International journal of pharmaceutical sciences and research. 7 (2016) 228.

31. S. K. Kashaw, et al., Development of 3D-QSAR models in cyclic ureidobenzenesulfonamides: human $\hat{\mathrm{I}}^{2} 3$-Adrenergic receptor agonist. Bioorganic \& medicinal chemistry letters. 13 (2003) 2481-2484.

32. E. Verma, et al., Mechanistic Approach to Explore Isoniazid Derivatives as Antitubercular Agents Using KNN-MF Based-QSAR Analysis, Pharmacophore Modeling and Molecular Docking. Current Drug Therapy. 12 (2017) 97-114.

33. J. R. Brown, et al., The structure activity relationship of urea derivatives as anti-tuberculosis agents. Bioorganic \& medicinal chemistry. 19 (2011) 5585-5595.

34. VLifeMDS: Molecular Design Suite, version 4.4, 2010, VLife Sciences Technologies Pvt. Ltd., Pune, India.

35. PHASE, version 9.3, 2013, Schrodinger, LLC, New York.

36. GOLD (Geneic Algorithm for Ligand Docking), version 5.2, 2015, GOLD CCDC Software Ltd., United Kingdom.

37. D. A. Case, et al., The Amber biomolecular simulation programs. Journal of computational chemistry. 26 (2005) 1668-1688.

38. H. Mehta, et al., Pharmacophore mapping and 3D-QSAR analysis of Staphylcoccus aureus Sortase a inhibitors. Der Pharma Chemica. 4 (2012) 1776-1784.

39. E. C. Ibezim, et al., Computer-aided linear modeling employing QSAR for drug discovery. Scientific Research and Essays. 4 (2009) 1559-1564.

40. A. K. Debnath, Quantitative structure-activity relationship (QSAR) paradigm--Hansch era to new millennium. Mini reviews in medicinal chemistry. 1 (2001) 187-195.

41. S. L. Dixon, et al., PHASE: a new engine for pharmacophore perception, 3D-QSAR model development, and 3D database screening: 1 . Methodology and preliminary results. Journal of computer-aided molecular design. 20 (2006) 647-671.

42. P. Kaur, V. Sharma and V. Kumar, Pharmacophore Modelling and 3D-QSAR Studies on Nð $\square \ddot{Y}^{\circ}-$ Phenylpyrazinones as Corticotropin-Releasing Factor 1 Receptor Antagonists. International journal of medicinal chemistry. 2012 (2012)

43. H. M. Berman, et al., The Protein Data Bank. Nucleic Acids Research. 28 (2000) 235-242.

44. J. W. Liebeschuetz, J. C. Cole and O. Korb, Pose prediction and virtual screening performance of GOLD scoring functions in a standardized test. Journal of computer-aided molecular design. 26 (2012) 737-748.

45. C. Kaur, S. Dhiman and H. Singh, Synthesis, screening and docking studies of benzochromone derivatives as xanthine oxidase inhibitors. J Chem Pharm Res. 7 (2015) 127-136.

46. V. Kumar and M. E. Sobhia, Molecular dynamics-based investigation of InhA substrate binding loop for diverse biological activity of direct InhA inhibitors. Journal of Biomolecular Structure and Dynamics. 34 (2016) 2434-2452.

47. V. Kumar and M. E. Sobhia, Molecular dynamics assisted mechanistic study of isoniazid-resistance against Mycobacterium tuberculosis InhA. PloS one. 10 (2015) e0144635.

48. V. Hornak, et al., Comparison of multiple Amber force fields and development of improved protein backbone parameters. Proteins: Structure, Function, and Bioinformatics. 65 (2006) 712-725. 
49. D. Studio, version 2.5; Accelrys. Inc., San Diego, CA. 92121 (2009)

50. W. D. Cornell, et al., A second generation force field for the simulation of proteins, nucleic acids, and organic molecules. Journal of the American Chemical Society. 117 (1995) 5179-5197.

51. J. Wang, et al., Development and testing of a general amber force field. Journal of computational chemistry. 25 (2004) 1157-1174.

52. W. L. Jorgensen, et al., Comparison of simple potential functions for simulating liquid water. The Journal of chemical physics. 79 (1983) 926-935.

53. W. Van Gunsteren and H. Berendsen, Algorithms for macromolecular dynamics and constraint dynamics. Molecular Physics. 34 (1977) 1311-1327.

54. T. Darden, D. York and L. Pedersen, Particle mesh Ewald: An N • $\log (\mathrm{N})$ method for Ewald sums in large systems. The Journal of chemical physics. 98 (1993) 10089-10092.

55. A. W. Götz, et al., Routine microsecond molecular dynamics simulations with AMBER on GPUs. 1. Generalized born. Journal of chemical theory and computation. 8 (2012) 1542-1555.

56. W. Humphrey, A. Dalke and K. Schulten, VMD: visual molecular dynamics. Journal of molecular graphics. 14 (1996) 33-38.

57. D. R. Roe and T. E. Cheatham III, PTRAJ and CPPTRAJ: software for processing and analysis of molecular dynamics trajectory data. Journal of chemical theory and computation. 9 (2013) 3084-3095.

58. B. A. Reva, A. V. Finkelstein and J. Skolnick, What is the probability of a chance prediction of a protein structure with an rmsd of 6 å? Folding and Design. 3 (1998) 141-147.

59. L. Yang, G. Song and R. L. Jernigan, Comparisons of experimental and computed protein anisotropic temperature factors. Proteins: Structure, Function, and Bioinformatics. 76 (2009) 164-175.

60. T. Hou, et al., Assessing the performance of the molecular mechanics/Poisson Boltzmann surface area and molecular mechanics/generalized Born surface area methods. II. The accuracy of ranking poses generated from docking. Journal of computational chemistry. 32 (2011) 866-877.

61. T. Hou, et al., Characterization of domain-peptide interaction interface: prediction of SH3 domainmediated protein-protein interaction network in yeast by generic structure-based models. Journal of proteome research. 11 (2012) 2982-2995.

62. H. Gohlke, C. Kiel and D. A. Case, Insights into protein-protein binding by binding free energy calculation and free energy decomposition for the Ras-Raf and Ras-RalGDS complexes. Journal of molecular biology. 330 (2003) 891-913.

63. V. Kumar and M. E. Sobhia, Characterisation of the flexibility of substrate binding loop in the binding of direct InhA inhibitors. International journal of computational biology and drug design. 6 (2013) 318-342.

64. V. Kumar and M. E. Sobhia, Insights into the bonding pattern for characterizing the open and closed state of the substrate-binding loop in Mycobacterium tuberculosis InhA. Future medicinal chemistry. 6 (2014) 605-616.

65. V. Kumar, et al., Identification of hotspot regions of MurB oxidoreductase enzyme using homology modeling, molecular dynamics and molecular docking techniques. Journal of molecular modeling. 17 (2011) 939-953.

66. A. Arvind, et al., Homology modeling, molecular dynamics and inhibitor binding study on MurD ligase of Mycobacterium tuberculosis. Interdisciplinary Sciences, Computational Life Sciences. 4 (2012) 223.

67. M. Awale, et al., Homology modeling and atomic level binding study of Leishmania MAPK with inhibitors. Journal of molecular modeling. 16 (2010) 475-488.

68. N. Lall, et al., In vitro and In vivo antimycobacterial, hepatoprotective and immunomodulatory activity of Euclea natalensis and its mode of action. Journal of ethnopharmacology. 194 (2016) 740-748.

69. V. Kumar, et al., Structure-function prediction of $\alpha 2 \mathrm{~A}-, \alpha 2 \mathrm{~B}-$, and $\alpha 2 \mathrm{C}$-adrenoceptors using homology model assisted antagonist binding study. Medicinal Chemistry Research. 23 (2014) 735-746.

70. N. Lall, et al., Insights into tyrosinase inhibition by compounds isolated from Greyia radlkoferi Szyszyl using biological activity, molecular docking and gene expression analysis. Bioorganic \& medicinal chemistry. 24 (2016) 5953-5959. 
Appendix A. Supplementary data

Table S1. Correlation matrix for the descriptors contributing to the 2D QSAR model

\begin{tabular}{|l|l|l|l|l|l|l|l|}
\hline & Chi1 & K2alpha & SsssNcount & SdOcount & SssOE & T_2_N_7 & SCORE \\
\hline Chi1 & 1 & & & & & & 5 \\
\hline K2alpha & 0.110904 & 1 & & & & & 5 \\
\hline SsssNcount & 0.343417 & -0.063986 & 1 & & & & 5 \\
\hline SdOcount & 0.177054 & -0.003655 & -0.025048 & 1 & & & 5 \\
\hline SssOEcount & 0.065343 & -0.194961 & -0.104268 & 0.205061 & 1 & & 5 \\
\hline T_2_N_7 & 0.331234 & 0.304349 & -0.10829 & 0.219269 & -0.091624 & 1 & 5 \\
\hline
\end{tabular}

Table S2. Uni-Column statistics for training set and test set

\begin{tabular}{lllllll}
\hline Set & $\begin{array}{l}\text { Column } \\
\text { Name }\end{array}$ & Average & Max & Min & $\begin{array}{l}\text { Standard } \\
\text { Deviation }\end{array}$ & Sum \\
& pIC50 & 8.4292 & 9.3970 & 5.7350 & 1.1156 & 269.7360 \\
Training & pIC50 & 7.7977 & 9.0000 & 6.2800 & 1.0209 & 62.3820 \\
& & & & & & \\
\hline
\end{tabular}


Table S3. Actual and predicted activity values of all compounds of dataset by 2D QSAR

\begin{tabular}{|l|l|l|l|l|l|l|l|}
\hline $\begin{array}{l}\text { Comp } \\
\text { No. }\end{array}$ & $\begin{array}{l}\text { Actual } \\
\mathrm{pIC}_{50}(\mathrm{nM})\end{array}$ & $\begin{array}{l}\text { Predicted } \\
\mathrm{pIC}(\mathrm{nM})\end{array}$ & Residual & $\begin{array}{l}\text { Comp } \\
\text { No. }\end{array}$ & $\begin{array}{l}\text { Actual } \\
\mathrm{pIC}_{50}(\mathrm{nM})\end{array}$ & $\begin{array}{l}\text { Predicted } \\
\mathrm{pIC}_{50}(\mathrm{nM})\end{array}$ & Residual \\
\hline $1 \mathrm{~s}$ & 9.397 & 9.34816 & 0.04884 & $21 \mathrm{~s}$ & 9.301 & 9.24819 & 0.05281 \\
\hline $2 \mathrm{~s}$ & 9.397 & 9.24588 & 0.15112 & $22 \mathrm{~s}$ & 9.397 & 9.40885 & -0.01185 \\
\hline $4 \mathrm{~s}$ & 9.397 & 9.14256 & 0.25444 & $24 \mathrm{~s}$ & 9.397 & 8.99658 & 0.40042 \\
\hline $5 \mathrm{~s}$ & 9.397 & 9.11637 & 0.28063 & $25 \mathrm{~s} *$ & 7.779 & 8.09404 & -0.31504 \\
\hline $6 \mathrm{~s}$ & 9.397 & 8.64166 & 0.75534 & $26 \mathrm{~s}$ & 7.823 & 7.82284 & 0.00016 \\
\hline $7 \mathrm{~s}$ & 9.397 & 9.20865 & 0.18835 & $27 \mathrm{~s}$ & 9.397 & 9.65179 & -0.25479 \\
\hline $8 \mathrm{~s}$ & 8.920 & 8.31177 & 0.60823 & $28 \mathrm{~s}$ & 9.397 & 9.75477 & -0.35777 \\
\hline $9 \mathrm{~s}$ & 7.250 & 7.86979 & -0.61979 & $29 \mathrm{~s}$ & 8.721 & 8.58728 & 0.13372 \\
\hline $10 \mathrm{~s}^{*}$ & 8.958 & 8.55426 & 0.40374 & $30 \mathrm{~s}$ & 8.431 & 8.92876 & -0.49776 \\
\hline $11 \mathrm{~s} *$ & 8.346 & 8.13893 & 0.20707 & $31 \mathrm{~s}$ & 8.318 & 8.49575 & -0.17775 \\
\hline $12 \mathrm{~s}$ & 9.301 & 9.75268 & -0.45168 & $32 \mathrm{~s}$ & 6.333 & 7.00886 & -0.67586 \\
\hline $13 \mathrm{~s}$ & 7.829 & 8.30608 & -0.47708 & $33 \mathrm{~s}$ & 7.419 & 7.51716 & -0.09816 \\
\hline $14 \mathrm{~s}$ & 8.346 & 9.10846 & -0.76246 & $34 \mathrm{~s}$ & 5.735 & 5.68621 & 0.04879 \\
\hline $15 \mathrm{~s}$ & 7.073 & 7.35176 & -0.27876 & $35 \mathrm{~s}$ & 7.844 & 7.25923 & 0.58477 \\
\hline $16 \mathrm{~s}$ & 7.616 & 7.51496 & 0.10104 & $36 \mathrm{~s}$ & 8.920 & 8.64702 & 0.27298 \\
\hline $17 \mathrm{~s} *$ & 7.211 & 7.50926 & -0.29826 & $37 \mathrm{~s} *$ & 6.280 & 6.28403 & -0.00403 \\
\hline $18 \mathrm{~s}$ & 9.397 & 8.57171 & 0.82529 & $38 \mathrm{~s}$ & 6.413 & 6.49391 & -0.08091 \\
\hline $19 \mathrm{~s} *$ & 8.193 & 8.36988 & -0.17688 & $39 \mathrm{~s}$ & 6.584 & 6.52753 & 0.05647 \\
\hline $20 \mathrm{~s} *$ & 9.000 & 8.807 & 0.193 & $40 \mathrm{~s} *$ & 6.615 & 6.62823 & -0.01323 \\
\hline
\end{tabular}

* indicates test set molecules 
Table S4. Actual and predicted activity values of all compounds of dataset by 3D QSAR

\begin{tabular}{|l|l|l|l|l|l|l|l|}
\hline $\begin{array}{l}\text { Comp } \\
\text { No. }\end{array}$ & $\begin{array}{l}\text { Actual } \\
\mathrm{pIC}_{50}(\mathrm{nM})\end{array}$ & $\begin{array}{l}\text { Predicted } \\
\mathrm{pIC} 50(\mathrm{nM})\end{array}$ & Residual & $\begin{array}{l}\text { Comp } \\
\text { No. }\end{array}$ & $\begin{array}{l}\text { Actual } \\
\mathrm{pIC}_{50}(\mathrm{nM})\end{array}$ & $\begin{array}{l}\text { Predicted } \\
\mathrm{pIC} C_{50}(\mathrm{nM})\end{array}$ & Residual \\
\hline $1 \mathrm{~s}$ & 9.397 & 8.82343 & 0.57357 & $21 \mathrm{~s}$ & 9.301 & 9.1585 & 0.1425 \\
\hline $2 \mathrm{~s}$ & 9.397 & 9.17855 & 0.21845 & $22 \mathrm{~s}$ & 9.397 & 9.397 & 0 \\
\hline $3 \mathrm{~s}$ & 9.397 & 9.17881 & 0.21819 & $23 \mathrm{~s}^{*}$ & 8.795 & 8.40349 & 0.39151 \\
\hline $4 \mathrm{~s}$ & 9.397 & 9.15824 & 0.23876 & $24 \mathrm{~s}$ & 9.397 & 8.82052 & 0.57648 \\
\hline $5 \mathrm{~s}$ & 9.397 & 8.82043 & 0.57657 & $25 \mathrm{~s}$ & 7.779 & 7.821 & -0.042 \\
\hline $6 \mathrm{~s}$ & 9.397 & 9.397 & 0 & $26 \mathrm{~s}$ & 7.823 & 8.193 & -0.37 \\
\hline $7 \mathrm{~s}$ & 9.397 & 9.397 & 0 & $27 \mathrm{~s}$ & 9.397 & 9.15853 & 0.23847 \\
\hline $8 \mathrm{~s}$ & 8.920 & 9.397 & -0.477 & $28 \mathrm{~s}$ & 9.397 & 9.397 & 0 \\
\hline $9 \mathrm{~s} *$ & 7.250 & 8.10485 & -0.85485 & $29 \mathrm{~s}$ & 8.721 & 9.15859 & -0.43759 \\
\hline $10 \mathrm{~s}$ & 8.958 & 8.61595 & 0.34205 & $30 \mathrm{~s}$ & 8.431 & 8.82358 & -0.39258 \\
\hline $11 \mathrm{~s}^{*}$ & 8.346 & 9.397 & -1.051 & $31 \mathrm{~s}$ & 8.318 & & - \\
\hline $12 \mathrm{~s}$ & 9.301 & 8.38851 & 0.91249 & $32 \mathrm{~s}$ & 6.333 & & - \\
\hline $13 \mathrm{~s}^{*}$ & 7.829 & 7.02478 & 0.80422 & $33 \mathrm{~s}$ & 7.419 & 7.90619 & -0.48719 \\
\hline $14 \mathrm{~s}$ & 8.346 & 8.91406 & -0.56806 & $34 \mathrm{~s}$ & 5.735 & 6.49848 & -0.76348 \\
\hline $15 \mathrm{~s}$ & 7.073 & 7.49478 & -0.42178 & $35 \mathrm{~s}$ & 7.844 & 9.17619 & -1.33219 \\
\hline $16 \mathrm{~s}$ & 7.616 & 7.4951 & 0.1209 & $36 \mathrm{~s}$ & 8.920 & 9.05891 & -0.13891 \\
\hline $17 \mathrm{~s}$ & 7.211 & 7.42573 & -0.21473 & $37 \mathrm{~s}^{*}$ & 6.280 & 6.80956 & -0.52956 \\
\hline $18 \mathrm{~s}$ & 9.397 & 9.397 & 0 & $38 \mathrm{~s}$ & 6.413 & 6.16024 & 0.25276 \\
\hline $19 \mathrm{~s}$ & 8.193 & 7.823 & 0.37 & $39 \mathrm{~s}$ & 6.584 & 6.07465 & 0.50935 \\
\hline $20 \mathrm{~s} *$ & 9.000 & 8.47954 & 0.52046 & $40 \mathrm{~s} *$ & 6.615 & 6.80956 & -0.19456 \\
\hline
\end{tabular}

* indicates test set of compounds

Table S5. Distances between pharmacophore features of hypothesis ADHHR.27

\begin{tabular}{ccc}
\hline Site1 & Site2 & Distance \\
\hline A1 & D2 & 3.131 \\
A1 & H5 & 6.537 \\
A1 & H6 & 6.563 \\
A1 & R8 & 4.047 \\
D2 & H5 & 6.712 \\
D2 & H6 & 7.846 \\
D2 & R8 & 5.092 \\
H5 & H6 & 2.764 \\
H5 & R8 & 2.763 \\
H6 & R8 & 2.765 \\
\hline
\end{tabular}


Table S6. Angles formed between pharmacophore features of hypothesis ADHHR.27

\begin{tabular}{|c|c|c|c|c|c|c|c|}
\hline Site1 & Site2 & Site3 & Angle & Site1 & Site2 & Site3 & Angle \\
\hline D2 & A1 & H5 & 79.5 & D2 & H5 & H6 & 103.8 \\
\hline D2 & A1 & H6 & 102.2 & D2 & H5 & R8 & 43.8 \\
\hline D2 & A1 & R8 & 89.4 & H6 & H5 & R8 & 60 \\
\hline H5 & A1 & H6 & 24.4 & A1 & H6 & D2 & 23 \\
\hline H5 & A1 & R8 & 13.4 & A1 & H6 & H5 & 77.3 \\
\hline H6 & A1 & R8 & 12.8 & A1 & H6 & R8 & 18.9 \\
\hline A1 & D2 & H5 & 73.2 & D2 & H6 & H5 & 56.2 \\
\hline A1 & D2 & H6 & 54.9 & D2 & H6 & R8 & 4.1 \\
\hline A1 & D2 & R8 & 52.6 & H5 & H6 & R8 & 60 \\
\hline H5 & D2 & H6 & 20 & A1 & R8 & D2 & 37.9 \\
\hline H5 & D2 & R8 & 22.1 & A1 & R8 & H5 & 146.8 \\
\hline H6 & D2 & R8 & 2.2 & A1 & R8 & H6 & 148.4 \\
\hline A1 & H5 & D2 & 27.3 & D2 & R8 & H5 & 114.1 \\
\hline A1 & H5 & H6 & 78.4 & D2 & R8 & H6 & 173.7 \\
\hline A1 & H5 & R8 & 19.8 & H5 & R8 & H6 & 60 \\
\hline
\end{tabular}


Table S7. Fitness scores of all the training and test set compounds dataset of training and test set molecules

\begin{tabular}{|c|c|c|c|c|c|}
\hline $\begin{array}{l}\text { Compound } \\
\text { name }\end{array}$ & $\begin{array}{l}\text { QSAR } \\
\text { Set }\end{array}$ & Pharm Set & Fitness & $\begin{array}{l}\text { Actual } \\
\text { activity }\end{array}$ & $\begin{array}{l}\text { Predicted } \\
\text { activity }\end{array}$ \\
\hline Comp 1s & training & active & 2.04 & 9.397 & 9.34 \\
\hline Comp 2s & training & active & 1.82 & 9.397 & 9.47 \\
\hline Comp 3s & training & active & 2.53 & 9.397 & 9.33 \\
\hline Comp 4s & training & active & 2.63 & 9.397 & 9.42 \\
\hline Comp 5s & training & active & 2.09 & 9.397 & 9.24 \\
\hline Comp 6s & training & active & 2.08 & 9.397 & 9.49 \\
\hline Comp 7s & training & active & 3 & 9.397 & 9.46 \\
\hline Comp 8s & test & & 2.9 & 8.921 & 9.09 \\
\hline Comp 9s & training & & 2.17 & 7.25 & 7.61 \\
\hline Comp 10s & test & & 1.92 & 8.959 & 8.61 \\
\hline Comp 11s & test & & 1.71 & 8.347 & 8.65 \\
\hline Comp 12s & training & active & 1.5 & 9.301 & 9.36 \\
\hline Comp 13s & test & & 1.75 & 7.83 & 8.42 \\
\hline Comp 14s & test & & 2.09 & 8.347 & 8.12 \\
\hline Comp 15s & test & & 1.66 & 7.074 & 7.62 \\
\hline Comp 16s & test & & 1.79 & 7.616 & 7.75 \\
\hline Comp 17s & training & & 1.86 & 7.212 & 7.23 \\
\hline Comp 18s & training & active & 1.66 & 9.398 & 9.43 \\
\hline Comp 19s & test & & 1.77 & 8.194 & 8.38 \\
\hline Comp 20s & training & active & 1.76 & 9 & 9.05 \\
\hline Comp 21s & training & active & 1.79 & 9.301 & 9.2 \\
\hline Comp 22s & training & active & 2.38 & 9.398 & 9.41 \\
\hline Comp 23s & test & & 2.04 & 8.796 & 9.66 \\
\hline Comp 24s & training & active & 2.8 & 9.398 & 9.35 \\
\hline Comp 25s & test & & 1.95 & 7.78 & 8.28 \\
\hline Comp 26s & training & & 2.42 & 7.824 & 7.72 \\
\hline Comp 27s & training & active & 1.86 & 9.398 & 9.6 \\
\hline Comp 28s & training & active & 2.39 & 9.398 & 9.27 \\
\hline Comp 29s & test & & 2.07 & 8.721 & 7.69 \\
\hline Comp 30s & training & & 1.79 & 8.432 & 8.42 \\
\hline Comp 31s & training & & 1.97 & 8.319 & 8.2 \\
\hline Comp 32s & training & inactive & 1.9 & 6.334 & 6.23 \\
\hline Comp 33s & test & & 1.45 & 7.419 & 7.4 \\
\hline Comp 34s & training & inactive & 1.33 & 5.736 & 5.85 \\
\hline Comp 35s & training & & 1.9 & 7.845 & 7.74 \\
\hline Comp 36s & training & & 1.5 & 8.921 & 8.88 \\
\hline Comp 37s & training & inactive & 1.64 & 6.28 & 6.39 \\
\hline Comp 38s & training & inactive & 1.63 & 6.413 & 6.39 \\
\hline Comp 39s & training & inactive & 1.61 & 6.584 & 6.6 \\
\hline Comp 40s & training & inactive & 1.62 & 6.615 & 6.47 \\
\hline
\end{tabular}

